"This document is the Accepted Manuscript version of a Published Work that appeared in final form in J. Org. Chem 2018, 83 (17), 9978-9990, copyright $@$ American Chemical Society after peer review and technical editing by the publisher. To access the final edited and published work see DOI: 10.1021/acs.joc.8b01372. This article may be used for non-commercial purposes in accordance with the ACS guidelines published at http://pubs.acs.org/page/policy/articlesonrequest/index.html]." 


\title{
Palladium-Catalyzed (Z)-Selective Allylation of Nitroalkanes: Access to Highly Functionalized Homoallylic Scaffolds
}

\author{
Àlex Cristòfol, ${ }^{\dagger, \ddagger}$ Eduardo C. Escudero-Adán ${ }^{\dagger}$ and Arjan W. Kleij*,+,\$
}

†Institute of Chemical Research of Catalonia (ICIQ), the Barcelona Institute of Science and Technology, Av. Països Catalans 16, 43007 - Tarragona, Spain, and łUniversitat Rovira i Virgili, Departament de Química Analítica i Química Orgànica, c/Marcel·lí Domingo, 1, 43007 Tarragona, Spain

${ }^{\S}$ Catalan Institute of Research and Advanced Studies (ICREA), Pg. Lluís Companys 23, 08010 - Barcelona, Spain
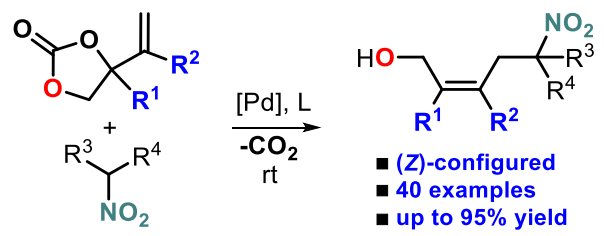

\begin{abstract}
Nitroalkanes undergo decarboxylative allylation in the presence of vinyl-substituted cyclic carbonates providing a wide variety of functionalized homoallylated compounds with exquisite stereocontrol. This Pd-mediated procedure features operational simplicity, versatile substrate combinations and also allows for the sequential introduction of different allyl groups in the nitroalkane scaffolds with high levels of stereocontrol through the intermediacy of a $(Z)$-configured palladacyclic intermediate. As far as we know, the developed protocol is the first general Pd-mediated methodology towards $(Z)$-configured homoallylic nitroalkanes with attractive functional group diversity.
\end{abstract}

\section{INTRODUCTION}

Stereoselective synthesis of highly functionalized tri- and tetrasubstituted olefin scaffolds still remains a highly challenging and attractive objective. ${ }^{1}$ Homoallylic nitroalkanes, an important subclass of alkenes, are versatile building blocks in organic synthesis and provide precursors to amines relevant to biologically active $\mathrm{N}$-containing compounds. ${ }^{2}$ Metal-catalyzed allylic alkylation reactions have become popular towards the preparation of functionalized olefins under mild reaction conditions. ${ }^{3}$ In this respect, Tsuji-Trost type allylation of nitroalkanes has received much attention since the resultant homoallylic products are precursors to their homoallylic amine congeners. ${ }^{4}$

While enantioselective allylations of nitroalkanes have been frequently investigated (Scheme 1a, route I) ${ }^{5}$ stereoselective allylations have been less studied (Scheme 1a, route II). ${ }^{6}$ In most reported cases, the resulting olefin unit in the homoallylic nitroalkane product is mostly disubstituted while having an $(E)$ configuration. In 1985, Hesse and coworker reported the allylation of 2-nitrocycloalkanones using substituted allylic carbonates affording rare examples of trisubstituted derivatives (Scheme 1a, lower part). ${ }^{7}$ However, either a stereoisomeric mixture or formation of expected stereochemistry $(E)$ was noted for the targeted products. Thus, despite notable progress the stereoselective construction of tri- and tetrasubstituted $(Z)$-configured homoallylic nitroalkanes using metal-catalyzed allylic substitution reactions remains undeveloped. An approach that successfully addresses these issues would expand on the variety of available homoallylic scaffolds that cannot be easily accessed through other known methodologies. ${ }^{1,8}$ Owing to the versatility of the nitro group, it can be anticipated that their use would be further amplified in synthetic chemistry.
Scheme 1. Previous Metal-Catalyzed Allylation of Nitroalkanes and Current Approach towards Tri- and Tetrasubstituted Homoallylic Nitroalkanes

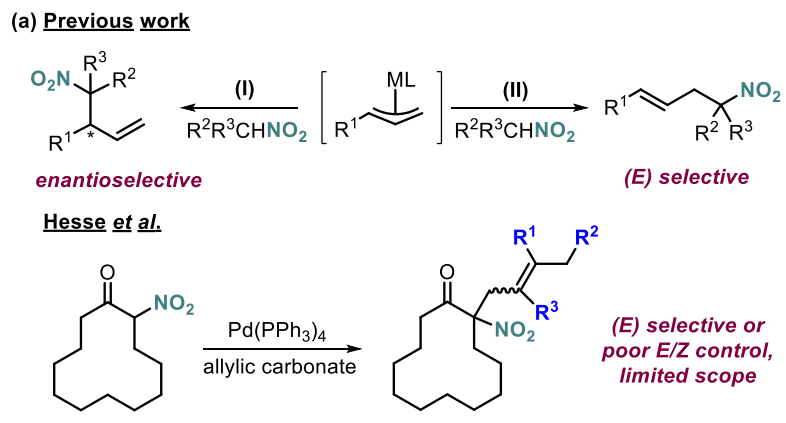

(b) This work

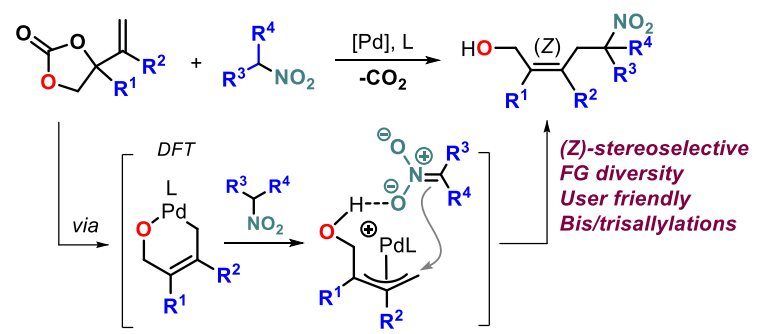

We recently reported that regio- and enantioselective Pdcatalyzed allylic substitutions of vinyl-substituted cyclic carbonates (VCCs) ${ }^{9}$ in the presence of $\mathrm{N}-,{ }^{10} \mathrm{O}-{ }^{11}$ and S-derived nucleophiles $^{12}$ are ligand-controlled transformations providing either linear or branched allylic compounds under high stereocontrol. Computational analysis afforded useful mechanistic insight into the origin of the stereocontrol, and moreover illustrated that a (Z)-palladacylic intermediate (which was supported 
by DFT analysis $)^{10 a}$ acts a key species incorporating a basic function ( $c f$., Scheme 1b) for the activation of pronucleophiles.

With this design element in mind, we envisioned that nitronate species should be easily formed from nitroalkanes in the presence of this formal $\mathrm{Pd}($ allyl) intermediate without the requirement of a basic additive. Suitable ligands should be able to bias the double bond configuration in the allylated nitroalkanes towards the desired stereoisomer. ${ }^{13}$ Importantly, the targeted (Z)-configured homoallylic compounds would incorporate additional functionality providing interesting product diversification potential from a wide array of homoallylic structures that are not easily accessible via other preparative routes.

Table 1. Optimized Reaction Conditions towards the Stereoselective Formation of Homoallylic Product 3a ${ }^{a}$

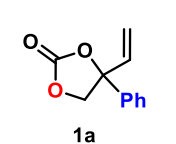

1a
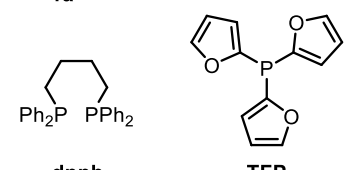

dppb

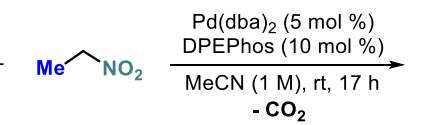

$-\mathrm{CO}_{2}$

L1

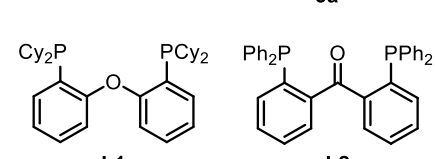

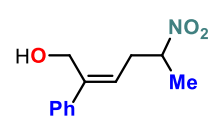

3a

\begin{tabular}{llll} 
entry & deviation $^{b}$ & $\begin{array}{l}\text { yield of } \\
\mathbf{3 a}(\%)^{c}\end{array}$ & $\mathrm{Z:E}^{d}$ \\
\hline 1 & none & $85(50)^{e}$ & $>99: 1$ \\
2 & $\mathrm{Pd}\left(\mathrm{PPh}_{3}\right)_{4} / \mathrm{THF}$ & 41 & $93: 7$ \\
3 & White catalyst/THF & 18 & $87: 13$ \\
4 & Toluene as solvent & 59 & $93: 7$ \\
5 & DMF as solvent & 76 & $98: 2$ \\
6 & dppb as ligand & 74 & $>99: 1$ \\
7 & TFP as ligand & 69 & $68: 32$ \\
8 & $\mathbf{L 1}$ as ligand & 53 & $>99: 1$ \\
9 & $\mathbf{L 2}$ as ligand & 56 & $87: 13$
\end{tabular}

${ }^{a}$ Reaction conditions: $0.20 \mathrm{mmol}$ of cyclic carbonate 1a, 0.30 mmol of nitroethane, $200 \mu \mathrm{L}$ of solvent, rt, open to air; the White catalyst is a bis-sulfoxide ligated $\mathrm{Pd}(\mathrm{OAc})_{2} .{ }^{b}$ Deviation from standard conditions. ${ }^{c} \mathrm{NMR}$ yield using mesitylene as an internal standard. ${ }^{d}$ Determined by ${ }^{1} \mathrm{H}$ NMR. ${ }^{e}$ Yield of isolated product.

\section{RESULTS AND DISCUSSION}

Screening Studies. Various conditions were screened towards the formation of the targeted $(Z)$-product 3a taking vinyl cyclic carbonate 1a and nitroethane as benchmark substrates. The nature of the Pd precursor, ligand, solvent, relative amount of nitroethane and catalyst loading were optimized (see Table 1 and Tables S1-S5, see the Supporting Information: SI). The optimized conditions reported in Table 1 gave the best NMR yield (Table 1 , entry $1 ; 85 \%$, isolated $50 \%)^{14}$ and stereoselectivity $(Z / E>99: 1)$ of homoallylic nitroalkane product 3a. The presence of other Pd-precursors, solvents or ligands (Table 1, entries 2-9) gave inferior results in terms of yield and/or stereocontrol, and are not discussed in detail here.
Scope in Nitroalkane Reagent. With these optimized conditions in hand, the scope of reaction partners was then examined, and first the nitroalkane reagent $(c f$., $\mathbf{2} \mathbf{a}-\mathbf{2 h}$, see SI for details on the preparation of the non-commercial examples) was varied (Scheme 2; note that isolated yields are reported) providing access to functional homoallylic nitroalkanes $\mathbf{3 a - 3 q}$ in excellent (except for $3 \mathrm{e})^{15}$ stereoselectivity. Apart from simple alkyl or aryl groups $\left(\mathrm{R}^{1}\right.$ and $\left.\mathrm{R}^{2}\right)$ in the nitroalkane reagent $(c f$., synthesis of $\mathbf{3 a}-\mathbf{d}$ ), also synthetically more attractive functionalities could be readily introduced into the allylated targets including alcohol (3e), alkyl chloride (3f), aryl sulfone (3g), ester $(\mathbf{3 h}, \mathbf{3 k})$, indole (3i), aromatic amine (3j), furan (3l), (dithio)acetal (3m-n) and imide (3o) groups. Most of these products were isolated in moderate to high yields, and apart from the desired compound typically bisallylated nitroalkanes ${ }^{16}$ and minor amounts $(<5 \%)$ of a vinyl carbonate cross-coupled derivative ${ }^{17}$ were noted. The presence of a free alcohol unit in the nitroalkane reagent leading to product $3 \mathbf{e}(Z / E=36: 64)$ is not welltolerated as illustrated by a significant lower stereoselectivity. This may be ascribed to undesired interaction of the alcohol with the intermediate (allyl)Pd species, and adversely influencing the stereochemical course of the allylation process.

Scheme 2. Variation of Nitroalkane Reagent to afford Homoallylic Compounds 3a-3q
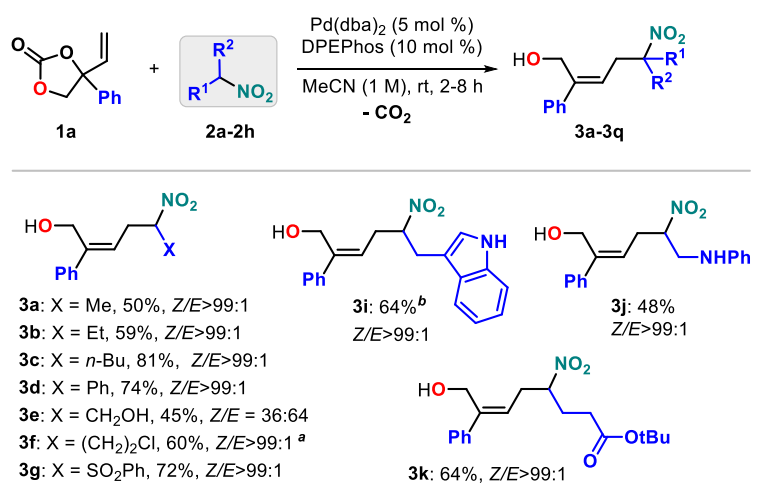
3h: $\mathrm{X}=\mathrm{CO}_{2} \mathrm{Et}, 80 \%$, Z/E>99:1
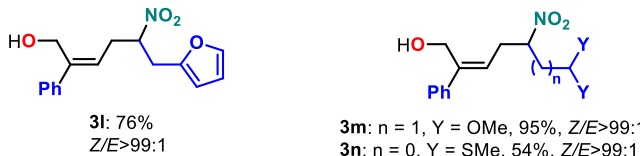

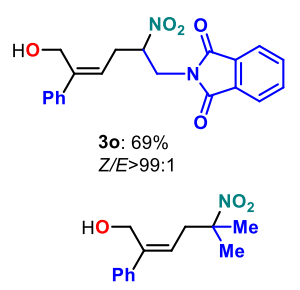

3p: $21 \%, Z / E>99: 1^{c}$ $83 \%(D B U), Z / E>99: 1^{c}$

$3 n: n=0, Y=S M e, 54 \%, Z / E>99: 1$

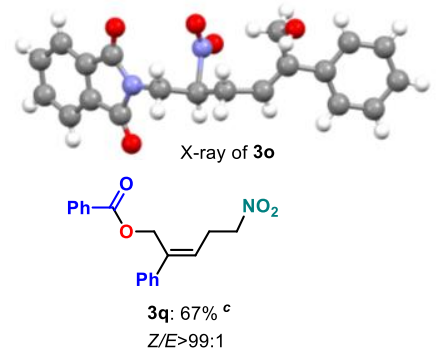

Conditions: cyclic carbonate $(1.0 \mathrm{mmol})$, nitroalkane ( 1.5 equiv.), $\mathrm{rt}, 2-8 \mathrm{~h}$, open to air. ${ }^{a} 0.23 \mathrm{mmol}$ scale. ${ }^{b} 0.58 \mathrm{mmol}$ scale. ${ }^{c} 0.20 \mathrm{mmol}$ scale. 
Apart from various primary nitroalkanes, also a more challenging secondary derivative could be allylated though with much lower efficiency (3p; $21 \%$ yield). To improve the formation of $\mathbf{3 p}$, the use of a base (DBU; 1,8-diazabicyclo[5.4.0] undec-7-ene) markedly improved the yield to $83 \%$, showing that the reactivity of the nitronate anion may be influenced by the counter cation. ${ }^{18}$

Finally, when benzoyl nitromethane was employed, we observed a post-synthetic shift of the phenyl ketone group to the alcohol unit resulting in the formation of the phenyl ester based allylated nitroalkane 3q isolated in $67 \%$ yield. ${ }^{19}$ The assigned stereochemistry of all products was fully consistent with their 2D NMR data ( see $^{1} \mathrm{H}^{-1} \mathrm{H}$ NOESY NMR for 3d; SI), and in the case of 3o, it was further confirmed by X-ray diffraction (see inset in Scheme 2 and SI).

\section{Scheme 3. Variation of the VCC Reagent to afford Homoallylic Compounds 4a-4n}
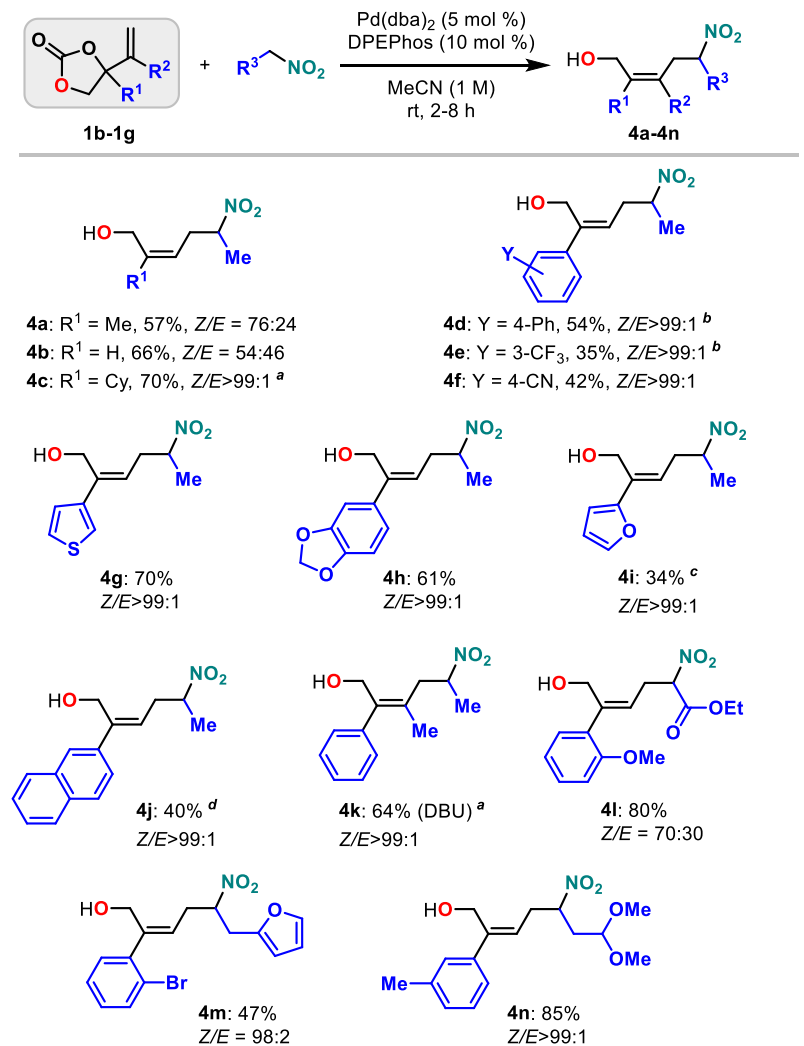

Conditions: cyclic carbonate ( $1.0 \mathrm{mmol})$, nitroalkane (1.5 equiv.), $\mathrm{rt}, 2-8 \mathrm{~h}$, open to air. Note that formally product $4 \mathrm{i}$ has an $(E)$-configuration, but has the same spatial orientation around the olefin unit as the other products. ${ }^{a} 0.20 \mathrm{mmol}$ scale. ${ }^{b}$ The reaction in the presence of DBU (10 mol \%) showed no improvement ( $32 \%$ isolated, Z/E $>99: 1) .{ }^{c} 0.46$ mmol scale. ${ }^{d} 0.22 \mathrm{mmol}$ scale.

Scope in VCC Reagent. Next, the VCC was varied using a selection of nitroalkanes as coupling partners and this afforded the homoallylated derivatives $\mathbf{4 a}-\mathbf{4 n}$ (Scheme 3). Apart from those cases where the VCC reagent was minimally substituted or having ortho-substituents in the aryl groups ( $c f$. , the synthesis of $\mathbf{4 a}-\mathbf{b}, \mathbf{4 l}$ with $\mathbf{4 m}$ being an exception), exclusive formation of the $(Z)$ isomer was noted. Alkyl-substituted VCCs show an apparent size-influence on the stereoselectivity of the reaction with the presence of larger groups such as cyclohexyl ( $c f$. , synthesis of $\mathbf{4 c}$ ) providing much higher $Z / E$ control. We previously reported the formal hydration of similar (allyl)Pd intermediates obtained after decarboxylation of $\mathrm{VCCs}^{11 \mathrm{a}}$ giving rise to $(Z)$ butene-1,4-diol formation. This latter work showed that hyper conjugation between the VCC aryl substituent and the Pd(allyl) fragment is crucial towards the overall reactivity and $(Z)$ stereobias. Interestingly, the hydration of alkyl-based Me- and cyclohexyl-substituted VCCs proved to be unproductive substrates in this hydration process, whereas in the present work the use of nitroalkanes as pronucleophiles was feasible towards product formation. Thus, both electronic as well as steric effects are operative in these kind of allylic transformations, and the type of nucleophile also plays an important role.

Both substituted aryl- and alkyl-substituted VCCs $\left(\mathrm{R}^{1}\right.$ and $\left.\mathrm{R}^{2} ; \mathbf{4 a}-\mathbf{4 f}\right)$ could be used, though the presence of electron-poor aryl groups lowered the yields of the homoallylic targets $(4 \mathbf{e}-$ 4f). Various heterocyclic groups $(\mathbf{4 g}-\mathbf{4 i})$ were also readily incorporated, though the isolated yield of $4 \mathbf{i}$ was low (34\%) due to the instability of the carbonate reagent. Other combinations of substituted VCCs and nitroalkanes were also probed furnishing the allylated nitroalkanes $\mathbf{4} \mathbf{j}-\mathbf{4 n}$ in up to $85 \%$ yield. Notably, product $4 \mathbf{k}$ (64\%, using DBU as additive) comprises a tetrasubstituted double bond, and stereoselective formation of such compounds with four different substituents is generally difficult. ${ }^{1}$

Scheme 4. Stereoselective Synthesis of Mono-, Bis- and Trisallylated Compounds.

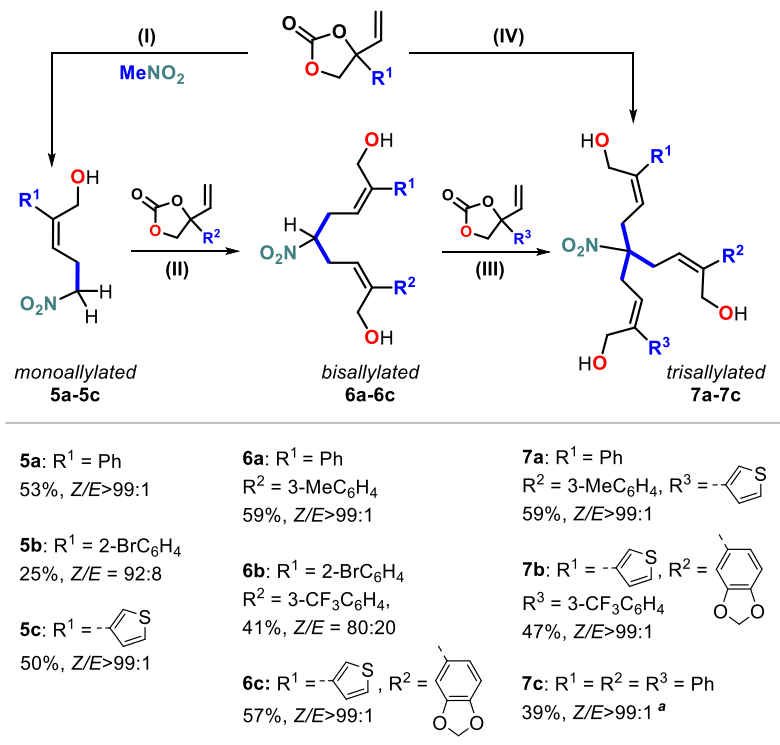

Conditions: (I) cyclic carbonate $(1.0 \mathrm{mmol}),[\mathrm{Pd}(\mathrm{allyl}) \mathrm{Cl}]_{2}(2.5 \mathrm{~mol} \%)$, DPEPhos (10 $\mathrm{mol} \%), \mathrm{CH}_{3} \mathrm{NO}_{2}(1.0 \mathrm{~mL}$ ), rt, open to air; (II) cyclic carbonate (1.0 equiv.), nitroalkane (1.5 equiv.), $\mathrm{Pd}(\mathrm{dba})_{2}(5.0 \mathrm{~mol} \%)$, DPEPhos $(10 \mathrm{~mol} \%), \operatorname{MeCN}(1 \mathrm{M})$, rt, open to air; (III) cyclic carbonate (1.0 equiv.), nitroalkane (1.5 equiv.), $\mathrm{Pd}(\mathrm{dba})_{2}(5.0 \mathrm{~mol} \%)$, DPEPhos (10 mol \%), DBU (10 mol \%), MeCN (1 M), rt, open to air; (IV) cyclic carbonate ( 3.1 equiv.), nitromethane ( 1.0 equiv.), Pd(dba) $)_{2}(5.0 \mathrm{~mol} \%$ ), DPEPhos (10 $\mathrm{mol} \%)$, DBU $(10 \mathrm{~mol} \%), \mathrm{MeCN}(1 \mathrm{M})$, rt, open to air. ${ }^{a}$ The reaction was repeated with DBU (30 mol \%) without improvement of the yield (40\%, Z/E>99:1). 
Synthesis of Multi-Allylated Nitroalkanes. Multiple allylation was then also investigated to see whether the stereoselectivity could be retained upon introduction of different allyl groups (Scheme 4). It was necessary to devise new reaction conditions for the synthesis of mono-allylated products $\mathbf{5 a}-\mathbf{c}$, as bisallylation was more competitive compared to the use of other primary nitroalkanes (see Tables S6-S8, SI). After finding suitable reaction conditions, nitroalkanes $\mathbf{5 a - 5 c}$ were prepared under excellent stereocontrol (Scheme 4, Route I). Subsequent allylation using standard conditions gave hetero-bisallylated nitroalkanes $6 \mathbf{a}$ and $\mathbf{6 c}$ with high $Z / E$ ratios while for $\mathbf{6 b}$ a lower degree of stereocontrol was noted (Scheme 4, Route II).

Hetero-trisallylation also proved be feasible ( $c f ., 7 \mathbf{a}$ and $\mathbf{7 b}$ ) and despite the higher steric demand of the intermediate carbon nucleophile (Scheme 4, Route III), excellent levels of stereocontrol were observed. Moreover, homo-trisallylated product 7c could be directly prepared in one-step from nitromethane with exquisite stereoselectivity (Scheme 4, Route IV). These results underpin the robust nature of these stereocontrolled conversions towards more elaborate functional scaffolds.

\section{Scheme 5. Synthesis of highly Substituted Homoallylic} Amines 8a and 8b.

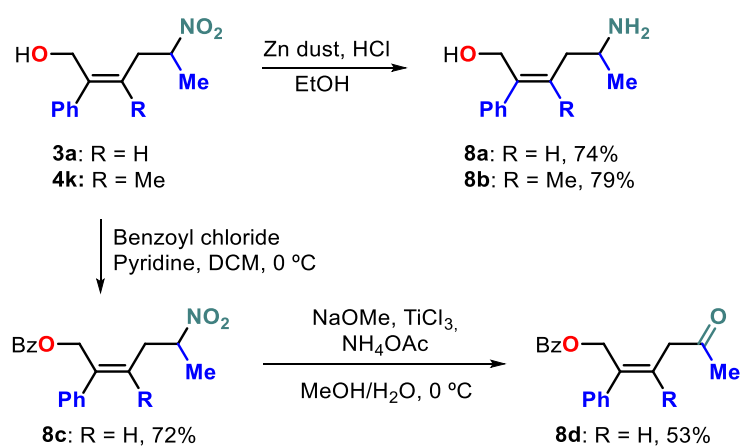

The allylation process could be easily scaled up $(6 \mathrm{mmol}$ of VCC used, $1.14 \mathrm{~g}$ ) using the same experimental procedure thus allowing to obtain larger amounts of homoallylic nitroalkane $\mathbf{3 a}$ without affecting the efficiency of the process $(62 \%$ yield, $Z / E$ $>99: 1$ ). The conversion of homoallylic nitroalkanes $\mathbf{3 a}$ and $\mathbf{4 k}$ into their homoallylic amines $\mathbf{8 a}$ and $\mathbf{8 b}$ (Scheme 5) was straightforward and these compounds were isolated in good yields. As far as we know, the synthesis of $\mathbf{8 b}$ represents the first example of a $(Z)$ configured homoallylic amine incorporating a tetrasubstituted double bond. Further to this, a combined protection/Nef reaction sequence (see the Experimental Section for details) leading to ketone $\mathbf{8 d}$ illustrates that elusive $\beta, \gamma$-unsaturated (Z)-configured ketones can be easily accessed without any observed double bond isomerization.

\section{- CONCLUSION}

In summary, a general ( $Z$ )-stereoselective methodology for highly substituted homoallylic nitroalkanes is reported that features attractive functional group diversity and operational simplicity. These ( $Z$ )-homoallylic compounds can be prepared from readily accessible starting materials, which were also shown to offer entries towards bis- and trisallylated functional homoallylic scaffolds while maintaining excellent stereocontrol.

\section{- EXPERIMENTAL SECTION}

General methods. Air and water-sensitive reactions were carried out in flame-dried glassware under an argon atmosphere using standard Schlenk manifold techniques. Reactions were monitored by TLC or ${ }^{1} \mathrm{H}$ NMR. TLC was carried out on 0.25 mm Merck aluminum backed sheets coated with 60 F254 silica gel. Visualization of the silica plates was achieved using a UV lamp $(\lambda=254 \mathrm{~nm})$ and/or by heating plates that were dipped in a $\mathrm{KMnO}_{4}$ or ceric ammonium molybdate stains. Flash chromatography was carried out on Sigma-Aldrich silica gel 60 (70230 mesh) using the indicated eluent system. In the screening phase, the internal standard was added after the reaction. Hereafter, an aliquot of the resulting mixture was taken and the yield was determined by means of ${ }^{1} \mathrm{H}$ NMR spectroscopy using $\mathrm{CDCl}_{3}$ as the solvent.

Commercially available reagents and solvents were purchased from Sigma-Aldrich, TCI, Strem Chemicals, Abcr $\mathrm{GmbH}$, Acros Organics or Alfa Aesar, and were used without further purification. Zinc dust was activated using a previously reported procedure. ${ }^{20}$

Solvents were dried using an Innovative Technology PURE SOLV solvent purification system. ${ }^{1} \mathrm{H}$ NMR, ${ }^{13} \mathrm{C}$ NMR, DEPT90, DEPT-135, and related 2D NMR (gCOSY90, gHMQC, gHMBC and gNOESY) spectra were recorded at room temperature on a Bruker AV-300, AV-400 or AV-500 spectrometer and referenced to their residual deuterated solvent signals. ${ }^{19} \mathrm{~F}$ NMR spectra were recorded on a Bruker AV-400. Coupling constants $(J)$ are reported in Hertz with the following splitting abbreviations: $\mathrm{s}=$ singlet, $\mathrm{d}=$ doublet, $\mathrm{t}=$ triplet, $\mathrm{q}=$ quadruplet, quint $=$ quintet, sextet $=$ sext, br $=$ broad and app $=$ apparent. All reported NMR values are given in parts per million (ppm). FTIR measurements were carried out on a Bruker Optics FTIR Alpha spectrometer. Mass spectrometric and X-ray analyses were performed by the Research Support Group at ICIQ.

Vinyl cyclic carbonate synthesis. The following cyclic carbonates were prepared according to a previously reported literature: ${ }^{10 a}$ 4-methyl-4-vinyl-1,3-dioxolan-2-one, 4-cyclohexyl4-vinyl-1,3-dioxolan-2-one, 4-(naphthalen-2-yl)-4-vinyl-1,3dioxolan-2-one, 4-([1,1'-biphenyl]-4-yl)-4-vinyl-1,3-dioxolan2-one, 4-(furan-2-yl)-4-vinyl-1,3-dioxolan-2-one, 4-(thiophen3-yl)-4-vinyl-1,3-dioxolan-2-one and 4-(benzo[d][1,3]dioxol5-yl)-4-vinyl-1,3-dioxolan-2-one. Otherwise, the cyclic carbonates were prepared following a previously reported literature procedure with minor modifications. ${ }^{21}$

Step 1: In a flame-dried round-bottomed flask, organocatalyst $\mathbf{A}(10 \mathrm{~mol} \%)$ and paraformaldehyde (3.0 equiv.) were suspended in dry THF $(0.25 \mathrm{M})$ under Ar. Then, the aldehyde $(1.0$ equiv.) and DIPEA (20 mol \%) were added, respectively. The mixture was stirred at $60^{\circ} \mathrm{C}$ (oil bath) for $24 \mathrm{~h}$. Hereafter, the mixture was concentrated in vacuo and the residue was purified by flash chromatography on silica gel to afford the corresponding hydroxymethyl ketone.

Step 2: In a flame-dried Schlenk flask, the corresponding hydroxymethyl ketone (1.0 equiv.) was dissolved in dry THF $(0.4 \mathrm{M})$ under Ar. The solution was cooled to $0^{\circ} \mathrm{C}$ (ice-water bath) and vinyl magnesium bromide (2.5 equiv., $1.0 \mathrm{M}$ in THF) was added dropwise over 20 minutes. After the addition, the 
mixture was allowed to warm to room temperature and was stirred for $2.5-5 \mathrm{~h}$. The reaction was quenched at $0^{\circ} \mathrm{C}$ (ice-water bath) with $\mathrm{NH}_{4} \mathrm{Cl}$ (aq, sat) and the phases were separated. The aqueous phase was extracted with EtOAc. The combined organic layers were washed with brine, dried over $\mathrm{MgSO}_{4}$, filtered and concentrated in vacuo. The crude product was used in the next step without further purification.

Step 3: In a flame-dried Schlenk flask, the crude product from step 2 was dissolved in dry $\mathrm{CH}_{2} \mathrm{Cl}_{2}(0.33 \mathrm{M})$ under $\mathrm{Ar}$. Then, anhydrous pyridine (6.0 equiv.) was added, and the mixture was cooled to $-72{ }^{\circ} \mathrm{C}$ (dry ice-ethanol bath). Then, a solution of triphosgene ( 0.5 equiv.) in dry $\mathrm{CH}_{2} \mathrm{Cl}_{2}(0.5 \mathrm{M})$ was cannulated to the mixture dropwise over 20-30 minutes. After that, the mixture was stirred for $30 \mathrm{~min}$, and the reaction was monitored by TLC. If the reaction had not gone to completion, it was allowed to warm to room temperature and was stirred for further $30 \mathrm{~min}-2 \mathrm{~h}$. The reaction was quenched at $0^{\circ} \mathrm{C}$ (ice-water bath) with $\mathrm{NH}_{4} \mathrm{Cl}$ (aq, sat) and the phases were separated. The aqueous phase was extracted with $\mathrm{CH}_{2} \mathrm{Cl}_{2}$. The combined organic layers were washed with $1 \mathrm{M} \mathrm{HCl}, \mathrm{NaHCO}_{3}$ (aq, sat), brine, and were dried over $\mathrm{Na}_{2} \mathrm{SO}_{4}$, filtered and concentrated in vacuo. The crude product was purified by flash chromatography on silica gel to afford the corresponding cyclic carbonate. Note: the reported yields of the cyclic carbonates are always referred to combined steps 2 and 3.

4-Methyl-4-vinyl-1,3-dioxolan-2-one and cyclic carbonate 1a were prepared from their commercially available hydroxymethyl ketones. Cyclic carbonate $1 \mathrm{~g}$ was prepared using isopropenyl magnesium bromide. The organocatalyst (A: perchlorate salt of 3-mesityl-4,5,6,7-tetrahydrobenzo[d]thiazol-3ium) used in the first step was prepared according to a slightly modified literature procedure, ${ }^{22}$ the only change being that the products was purified by trituration using hot $i$ - $\mathrm{PrOH}\left(70{ }^{\circ} \mathrm{C}\right)$, instead of recrystallization from a mixture of $\mathrm{MeOH} / \mathrm{CH}_{2} \mathrm{Cl}_{2} / \mathrm{Et}_{2} \mathrm{O}$.

4-Phenyl-4-vinyl-1,3-dioxolan-2-one (1a). Compound 1a was prepared following the general procedure from 2-hydroxyacetophenone $(2.72 \mathrm{~g}, 20.0 \mathrm{mmol})$. The product was purified by flash chromatography on silica gel using EtOAc/cyclohexane (1:9) to afford the title compound ( $2.63 \mathrm{~g}, 69 \%$ yield) as a yellowish oil. ${ }^{1} \mathrm{H}$ NMR $\left(400 \mathrm{MHz}, \mathrm{CDCl}_{3}\right): \delta \mathrm{H} 7.46-7.34(\mathrm{~m}, 5 \mathrm{H})$, $6.16(\mathrm{dd}, J=17.2,10.7 \mathrm{~Hz}, 1 \mathrm{H}), 5.43(\mathrm{~d}, J=10.7 \mathrm{~Hz}, 1 \mathrm{H}), 5.42$ $(\mathrm{d}, J=17.2 \mathrm{~Hz}, 1 \mathrm{H}), 4.66(\mathrm{~d}, J=8.4 \mathrm{~Hz}, 1 \mathrm{H}), 4.58(\mathrm{~d}, J=8.4$ $\mathrm{Hz}, 1 \mathrm{H}) .{ }^{13} \mathrm{C} \mathrm{NMR}\left(101 \mathrm{MHz}, \mathrm{CDCl}_{3}\right): \delta 154.2,138.5,136.6$, $129.1,129.0,125.0,117.6,85.6,74.6$. Spectroscopic data are in agreement with published data. ${ }^{9 \mathrm{c}}$

4-(m-Tolyl)-4-vinyl-1,3-dioxolan-2-one $(\mathbf{1 b})$. Compound $\mathbf{1 b}$ was prepared following general procedure from $m$-tolualdehyde (1.82 $\mathrm{mL}, 15.0 \mathrm{mmol})$. The product was purified by flash chromatography on silica gel using $5 \%$ EtOAc/cyclohexane to afford the title compound ( $1.86 \mathrm{~g}, 75 \%$ yield) as a yellowish oil. ${ }^{1} \mathrm{H}$ NMR $\left(500 \mathrm{MHz}, \mathrm{CDCl}_{3}\right): \delta 7.31-7.27(\mathrm{~m}, 1 \mathrm{H}), 7.19-7.16$ (m, 2H), 7.13-7.10 (m, 1H), $6.15(\mathrm{dd}, J=17.2,10.8 \mathrm{~Hz}, 1 \mathrm{H})$, $5.42(\mathrm{~d}, J=17.2 \mathrm{~Hz}, 1 \mathrm{H}), 5.41(\mathrm{~d}, J=10.8 \mathrm{~Hz}, 1 \mathrm{H}), 4.64(\mathrm{~d}, J$ $=8.4 \mathrm{~Hz}, 1 \mathrm{H}), 4.56(\mathrm{~d}, J=8.4 \mathrm{~Hz}, 1 \mathrm{H}), 2.38(\mathrm{~s}, 3 \mathrm{H}) .{ }^{13} \mathrm{C} \mathrm{NMR}$ $\left(126 \mathrm{MHz}, \mathrm{CDCl}_{3}\right): \delta 154.2,139.0,138.5,136.7,129.7,129.0$, $125.5,122.0,117.5,85.6,74.7,21.6$. Spectroscopic data are in agreement with published data. ${ }^{11 \mathrm{a}}$

4-(3-(Trifluoromethyl)phenyl)-4-vinyl-1,3-dioxolan-2-one (1c). Compound 1c was prepared following the general procedure from 3-(trifluoromethyl)benzaldehyde $(2.0 \mathrm{~mL}, 15.0$ $\mathrm{mmol})$. The product was purified by flash chromatography on silica gel using $20 \%$ EtOAc/cyclohexane to afford the title compound (1.28 g, $51 \%$ yield) as a light orange oil. $R_{\mathrm{f}} 0.25$ (EtOAc/cyclohexane 2:8). IR (neat): 2924, 1801, 1481, 1446, 1414, 1378, 1332, 1251, 1167, 1122, 1055, 986, 940, 902, 805, 768, 701, $654 \mathrm{~cm}^{-1} .{ }^{1} \mathrm{H}$ NMR $\left(400 \mathrm{MHz}, \mathrm{CDCl}_{3}\right): \delta 7.70-7.64$ $(\mathrm{m}, 1 \mathrm{H}), 7.63-7.60(\mathrm{~m}, 1 \mathrm{H}), 7.60-7.55(\mathrm{~m}, 2 \mathrm{H}), 6.16(\mathrm{dd}, J=$ $17.2,10.8 \mathrm{~Hz}, 1 \mathrm{H}), 5.49$ (d, $J=10.8 \mathrm{~Hz}, 1 \mathrm{H}), 5.44$ (d, $J=17.2$ $\mathrm{Hz}, 1 \mathrm{H}), 4.71(\mathrm{~d}, J=8.6 \mathrm{~Hz}, 1 \mathrm{H}), 4.56(\mathrm{~d}, J=8.6 \mathrm{~Hz}, 1 \mathrm{H}) .{ }^{13} \mathrm{C}$ NMR (101 MHz, $\left.\mathrm{CDCl}_{3}\right): \delta 153.7,139.8,136.0,131.7$ (q, $J=$ $32.7 \mathrm{~Hz}), 129.9,128.4,126.0(\mathrm{q}, J=3.8 \mathrm{~Hz}), 122.0(\mathrm{q}, J=3.8$ $\mathrm{Hz}), 118.6,85.1,74.4 .{ }^{19} \mathrm{~F}$ NMR $\left(376 \mathrm{MHz}, \mathrm{CDCl}_{3}\right): \delta$-62.9. HRMS (ESI+/TOF) Calcd for $\mathrm{C}_{12} \mathrm{H}_{9} \mathrm{~F}_{3} \mathrm{O}_{3} \mathrm{Na}^{+}[\mathrm{M}+\mathrm{Na}]^{+}$: 281.0396. Found: 281.0400.

4-(2-Methoxyphenyl)-4-vinyl-1,3-dioxolan-2-one (1d). Compound 1d was prepared following the general procedure from $o$-anisaldehyde $(1.81 \mathrm{~mL}, 15.0 \mathrm{mmol})$. The product was purified by flash chromatography on silica gel using $20 \%$ EtOAc/cyclohexane to afford the title compound (1.66 g, $89 \%$ yield) as a yellowish oil. ${ }^{1} \mathrm{H}$ NMR $\left(400 \mathrm{MHz}, \mathrm{CDCl}_{3}\right): \delta 7.51$ $(\mathrm{dd}, J=7.6,1.7 \mathrm{~Hz}, 1 \mathrm{H}), 7.35$ (ddd, $J=8.2,7.5,1.7 \mathrm{~Hz}, 1 \mathrm{H})$, $7.02(\mathrm{td}, J=7.6,1.1 \mathrm{~Hz}, 1 \mathrm{H}), 6.94(\mathrm{dd}, J=8.2,1.1 \mathrm{~Hz}, 1 \mathrm{H})$, $6.23(\mathrm{dd}, J=17.1,10.7 \mathrm{~Hz}, 1 \mathrm{H}), 5.40(\mathrm{~d}, J=17.1 \mathrm{~Hz}, 1 \mathrm{H}), 5.25$ $(\mathrm{d}, J=10.7 \mathrm{~Hz}, 1 \mathrm{H}), 4.74(\mathrm{~d}, J=8.8 \mathrm{~Hz}, 1 \mathrm{H}), 4.54(\mathrm{~d}, J=8.8$ $\mathrm{Hz}, 1 \mathrm{H}), 3.85$ (s, 3H). ${ }^{13} \mathrm{C}$ NMR $\left(101 \mathrm{MHz}, \mathrm{CDCl}_{3}\right): \delta 154.9$, 154.3, 136.4, 130.1, 127.6, 125.7, 121.4, 115.6, 111.2, 84.8, 75.2, 55.6. Spectroscopic data are in agreement with published data. ${ }^{9 \mathrm{c}}$

4-(2-Bromophenyl)-4-vinyl-1,3-dioxolan-2-one (1e). Compound 1e was prepared following the general procedure from 2 bromobenzaldehyde $(1.80 \mathrm{~mL}, 15.0 \mathrm{mmol})$. The product was purified by flash chromatography on silica gel using 15-20\% EtOAc/cyclohexane to afford the title compound (1.92 g, $57 \%$ yield) as a yellowish oil. ${ }^{1} \mathrm{H}$ NMR $\left(500 \mathrm{MHz}, \mathrm{CDCl}_{3}\right): \delta 7.70$ $(\mathrm{dd}, J=7.8,1.7 \mathrm{~Hz}, 1 \mathrm{H}), 7.62(\mathrm{dd}, J=7.8,1.2 \mathrm{~Hz}, 1 \mathrm{H}), 7.41$ $(\mathrm{td}, J=7.8,1.2 \mathrm{~Hz}, 1 \mathrm{H}), 7.25(\mathrm{td}, J=7.8,1.9 \mathrm{~Hz}, 1 \mathrm{H}), 6.36(\mathrm{dd}$, $J=17.0,10.6 \mathrm{~Hz}, 1 \mathrm{H}), 5.40(\mathrm{~d}, J=10.6 \mathrm{~Hz}, 1 \mathrm{H}), 5.40(\mathrm{~d}, J=$ $17.0 \mathrm{~Hz}, 1 \mathrm{H}), 4.97(\mathrm{~d}, J=9.0 \mathrm{~Hz}, 1 \mathrm{H}), 4.73(\mathrm{~d}, J=9.0 \mathrm{~Hz}, 1 \mathrm{H})$. ${ }^{13} \mathrm{C}$ NMR $\left(126 \mathrm{MHz}, \mathrm{CDCl}_{3}\right): \delta 153.5,138.8,135.1,134.4$, 130.5, 128.2, 127.5, 119.1, 118.6, 85.6, 74.6. Spectroscopic data are in agreement with published data. ${ }^{9 \mathrm{c}}$

4-(2-Oxo-4-vinyl-1,3-dioxolan-4-yl)benzonitrile (1f). Compound 1f was prepared following the general procedure from 4cyanobenzaldehyde $(1.97 \mathrm{~g}, 15.0 \mathrm{mmol})$, except that dry THF was used as solvent instead of $\mathrm{CH}_{2} \mathrm{Cl}_{2}$ in step 3 because of solubility problems. The product was purified by flash chromatography on silica gel using 20-30\% EtOAc/cyclohexane to afford the title compound (277.9 $\mathrm{mg}, 24 \%$ yield) as a light orange solid. $R_{\mathrm{f}} 0.13$ (EtOAc/cyclohexane 2:8). IR (neat): 3103, 3055, 2985, 2923, 2231, 1794, 1610, 1507, 1473, 1418, 1389, 1320, $1213,1117,1063,985,949,841,760,740 \mathrm{~cm}^{-1} .{ }^{1} \mathrm{H}$ NMR $(400$ $\left.\mathrm{MHz}, \mathrm{CDCl}_{3}\right): \delta 7.77-7.68(\mathrm{~m}, 2 \mathrm{H}), 7.53-7.43(\mathrm{~m}, 2 \mathrm{H}), 6.12$ $(\mathrm{dd}, J=17.2,10.8 \mathrm{~Hz}, 1 \mathrm{H}), 5.46(\mathrm{~d}, J=10.8 \mathrm{~Hz}, 1 \mathrm{H}), 5.41(\mathrm{~d}$, $J=17.2 \mathrm{~Hz}, 1 \mathrm{H}), 4.70(\mathrm{~d}, J=8.7 \mathrm{~Hz}, 1 \mathrm{H}), 4.53(\mathrm{~d}, J=8.7 \mathrm{~Hz}$, $1 \mathrm{H}) .{ }^{13} \mathrm{C} \mathrm{NMR}\left(101 \mathrm{MHz}, \mathrm{CDCl}_{3}\right): \delta 153.4,143.5,135.6,132.9$, 125.8, 118.7, 118.0, 113.1, 84.9, 74.2. HRMS (ESI+/TOF) Calcd for $\mathrm{C}_{12} \mathrm{H}_{9} \mathrm{NO}_{3} \mathrm{Na}^{+}[\mathrm{M}+\mathrm{Na}]^{+}$: 238.0475. Found: 238.0483 .

4-Phenyl-4-(prop-1-en-2-yl)-1,3-dioxolan-2-one (1g). Compound $1 \mathrm{~g}$ was prepared following the general procedure from 2-hydroxyacetophenone ( $1.36 \mathrm{~g}, 10.0 \mathrm{mmol})$. The product was purified by flash chromatography on silica gel using $10 \%$ EtOAc/cyclohexane to afford the title compound (1.31 g, $64 \%$ 
yield) as a white solid. ${ }^{1} \mathrm{H}$ NMR $\left(500 \mathrm{MHz}, \mathrm{CDCl}_{3}\right): \delta 7.44$ $7.33(\mathrm{~m}, 5 \mathrm{H}), 5.19(\mathrm{~s}, 1 \mathrm{H}), 5.16(\mathrm{~s}, 1 \mathrm{H}), 4.79(\mathrm{~d}, J=8.5 \mathrm{~Hz}$, $1 \mathrm{H}), 4.63(\mathrm{~d}, J=8.5 \mathrm{~Hz}, 1 \mathrm{H}), 1.73(\mathrm{~s}, 3 \mathrm{H}) .{ }^{13} \mathrm{C} \mathrm{NMR}(126 \mathrm{MHz}$, $\left.\mathrm{CDCl}_{3}\right): \delta 154.1,143.0,138.5,129.0,125.1,114.1,88.1,73.2$, 18.6. Spectroscopic data are in agreement with published data. ${ }^{11 \mathrm{a}}$

Nitroalkane synthesis. 3-(2-Nitroethyl)- $1 H$-indole (2a), ${ }^{23}$ tert-butyl 4-nitrobutanoate $(\mathbf{2 b})^{24}, 2$-(2-nitroethyl)furan $(\mathbf{2 c}){ }^{25}$ $N$-(2-nitroethyl)aniline (2d), ${ }^{25}$ 1,1-dimethoxy-3-nitropropane $(\mathbf{2 e})^{26}$ and 1-chloro-3-nitropropane $(\mathbf{2 h})^{27}$ were prepared following previously reported protocols. Nitroalkanes $\mathbf{2 f}$ and $\mathbf{2 g}$ were prepared as indicated below.

$N$-(2-Nitroethyl)phthalimide $(2 f)$. The following procedure was adapted from a previously reported procedure. ${ }^{24 \mathrm{~b}}$ In an oven-dried $100 \mathrm{~mL}$ round-bottomed flask, $\mathrm{PPh}_{3}(4.72 \mathrm{~g}, 18.0$ $\mathrm{mmol})$ and imidazole $(1.38 \mathrm{~g}, 19.5 \mathrm{mmol})$ were dissolved in dry $\mathrm{CH}_{2} \mathrm{Cl}_{2}$ under Ar. The mixture was cooled to $0{ }^{\circ} \mathrm{C}$ (ice-water bath), and $\mathrm{I}_{2}$ was added. After stirring the mixture for 15 minutes, $N$-(2-hydroxyethyl)phthalimide $(2.87 \mathrm{~g}, 15.0 \mathrm{mmol})$ was added. The mixture was stirred at $0{ }^{\circ} \mathrm{C}$ for 30 minutes and then at room temperature for $4 \mathrm{~h}$. After that, the reaction was diluted with $\mathrm{H}_{2} \mathrm{O}$ and the layers were separated. The aqueous phase was extracted with $\mathrm{CH}_{2} \mathrm{Cl}_{2}$, and the combined organic layers were dried over $\mathrm{Na}_{2} \mathrm{SO}_{4}$, filtered and concentrated in vacuo. The crude product was purified by flash chromatography on silica gel using $10 \% \mathrm{EtOAc/hexane} \mathrm{to} \mathrm{afford} \mathrm{N}$-(2-iodoethyl)phthalimide as a white solid (4.19 g, $93 \%$ yield).

In a $100 \mathrm{~mL}$ round-bottomed flask, $N$-(2-iodoethyl)phthalimide $(3.77 \mathrm{~g}, 12.51 \mathrm{mmol})$ was suspended in $\mathrm{H}_{2} \mathrm{O}(25 \mathrm{~mL})$ and then, $\mathrm{AgNO}_{2}(7.70 \mathrm{~g}, 50.04 \mathrm{mmol})$ was added. The flask was wrapped with aluminum foil and the mixture was heated at $60{ }^{\circ} \mathrm{C}$ (oil-bath) for $6 \mathrm{~h}$. After that, the mixture was filtered through a pad of Celite, and the filtrate was extracted with EtOAc. The combined organic layers were washed with brine, dried over $\mathrm{MgSO}_{4}$, filtered and concentrated in vacuo. The crude product was purified by flash chromatography on silica gel using 20-30 \% EtOAc/cyclohexane to afford the title compound as a white solid (645 mg, $23 \%$ yield). $R_{\mathrm{f}} 0.35$ (EtOAc/cyclohexane 3:7). IR (neat): 3463, 3096, 3066, 3031, 3004, 2965, 2927, 1771, 1701, 1608, 1549, 1418, 1394, 1343, 1302, 1229, 1186, 1143, 1087, 1043, 1018, 961, 877, 806, 717 $\mathrm{cm}^{-1} .{ }^{1} \mathrm{H}$ NMR $\left(500 \mathrm{MHz}, \mathrm{CDCl}_{3}\right): \delta 7.91-7.84(\mathrm{~m}, 2 \mathrm{H}), 7.79-$ $7.72(\mathrm{~m}, 2 \mathrm{H}), 4.72(\operatorname{app~t}, J=6.1 \mathrm{~Hz}, 2 \mathrm{H}), 4.32($ app t, $J=6.1$ $\mathrm{Hz}, 2 \mathrm{H}) .{ }^{13} \mathrm{C} \mathrm{NMR}\left(126 \mathrm{MHz}, \mathrm{CDCl}_{3}\right): \delta 167.6,134.6,131.8$, 123.9, 72.3, 35.3. HRMS (ESI+/TOF) Calcd for $\mathrm{C}_{10} \mathrm{H}_{8} \mathrm{~N}_{2} \mathrm{O}_{4} \mathrm{Na}^{+}$ $[\mathrm{M}+\mathrm{Na}]^{+}:$243.0376. Found: 243.0374 .

(2-Nitroethane-1,1-diyl)bis(methylsulfane) (2g). The following procedure was adapted from a previously reported procedure. ${ }^{27}$ In a flame-dried $100 \mathrm{~mL}$ round-bottomed flask, $\mathrm{NaBH}_{4}$ (454 mg, $12.0 \mathrm{mmol}$ ) was dissolved in dry THF (11 mL) and dry $\mathrm{MeOH}(3.8 \mathrm{~mL})$ under Ar. The solution was cooled to $0{ }^{\circ} \mathrm{C}$ (ice-water bath) and a solution of 1,1-bis(methylthio)-2nitroethylene $(1.65 \mathrm{~g}, 10.0 \mathrm{mmol})$ in dry THF $(15 \mathrm{~mL})$ was added. The mixture was warmed to room temperature and was stirred for $24 \mathrm{~h}$. The reaction was quenched with $\mathrm{NH}_{4} \mathrm{Cl}$ (aq, sat), was diluted with $\mathrm{H}_{2} \mathrm{O}$ and was extracted with EtOAc. The organic phase was washed with $\mathrm{H}_{2} \mathrm{O}$ and brine, dried over $\mathrm{Na}_{2} \mathrm{SO}_{4}$, filtered and concentrated in vacuo. The crude product was purified by flash chromatography on silica gel using $3 \%$ EtOAc/cyclohexane to afford the title compound as a colorless oil (768.5 mg, $46 \%$ yield). ${ }^{1} \mathrm{H}$ NMR (500 MHz, $\left.\mathrm{CDCl}_{3}\right): \delta 4.61$ $(\mathrm{d}, J=7.7 \mathrm{~Hz}, 2 \mathrm{H}), 4.36(\mathrm{t}, J=7.7 \mathrm{~Hz}, 1 \mathrm{H}), 2.19(\mathrm{~s}, 6 \mathrm{H}) .{ }^{13} \mathrm{C}$
NMR (126 MHz, $\left.\mathrm{CDCl}_{3}\right): \delta 77.9,50.2,13.2$. Spectroscopic data are in agreement with published data. ${ }^{28}$

General allylation procedure. In a $5 \mathrm{~mL}$ vial, cyclic carbonate (1.0 equiv.), Pd(dba) $)_{2}(5.0 \mathrm{~mol} \%)$, DPEPhos $(10 \mathrm{~mol} \%)$ and nitroalkane (1.5 equiv.) were dissolved in MeCN (1.0 M). The vial was sealed with a septum and a needle was placed through the septum to release gaseous $\mathrm{CO}_{2}$. The mixture was stirred for $2-8 \mathrm{~h}$ at $\mathrm{rt}$ open to air. After that, the solvent was evaporated with a stream of $\mathrm{N}_{2}$ and the residue was purified by flash chromatography on silica gel to afford the corresponding homoallylic nitroalkane. All homoallylic nitroalkanes apart from products $\mathbf{3 p}, \mathbf{4 k}, \mathbf{5 a - 5} \mathbf{c}$ and $\mathbf{7 a - 7} \mathbf{c}$ were prepared using this protocol.

(Z)-5-Nitro-2-phenylhex-2-en-1-ol (3a). Compound 3a was prepared following the general procedure from cyclic carbonate $1 \mathbf{a}(190.2 \mathrm{mg}, 1.0 \mathrm{mmol})$ and nitroethane. The product was purified by flash chromatography on silica gel using 20-30\% EtOAc/cyclohexane to afford the title compound (110.0 mg, 50 $\%$ yield) as a yellowish oil. $R_{\mathrm{f}} 0.31$ (EtOAc/cyclohexane $3: 7$ ). IR (neat): 3573, 3387, 3058, 2938, 1543, 1493, 1446, 1388, 1359, 1312, 999, 767, $697 \mathrm{~cm}^{-1} .{ }^{1} \mathrm{H}$ NMR (400 MHz, $\left.\mathrm{CDCl}_{3}\right): \delta$ $7.42-7.26(\mathrm{~m}, 5 \mathrm{H}), 5.74(\mathrm{dd}, J=8.2,7.2 \mathrm{~Hz}, 1 \mathrm{H}), 4.71$ (dqd, $J$ $=8.2,6.7,5.4 \mathrm{~Hz}, 1 \mathrm{H}), 4.61-4.50(\mathrm{~m}, 2 \mathrm{H}), 3.02(\mathrm{dt}, J=15.0$, $8.2 \mathrm{~Hz}, 1 \mathrm{H}), 2.74$ (ddd, $J=15.0,7.2,5.4 \mathrm{~Hz}, 1 \mathrm{H}), 1.62(\mathrm{~d}, J=$ $6.7 \mathrm{~Hz}, 3 \mathrm{H}), 1.52-1.46(\mathrm{~m}, 1 \mathrm{H}) .{ }^{13} \mathrm{C} \mathrm{NMR}\left(101 \mathrm{MHz}, \mathrm{CDCl}_{3}\right)$ : $\delta$ 143.6, 140.3, 128.7, 128.0, 126.7, 124.3, 83.3, 59.9, 34.0, 19.1. HRMS (ESI+/TOF) Calcd for $\mathrm{C}_{12} \mathrm{H}_{15} \mathrm{NO}_{3} \mathrm{Na}^{+}[\mathrm{M}+\mathrm{Na}]^{+}$: 244.0950. Found: 244.0944.

(Z)-5-Nitro-2-phenylhept-2-en-1-ol (3b). Compound $\mathbf{3 b}$ was prepared following the general procedure from cyclic carbonate 1a (190.2 mg, $1.0 \mathrm{mmol}$ ) and 1-nitropropane. The product was purified by flash chromatography on silica gel using 20 $\%$ EtOAc/cyclohexane to afford the title compound (138.2 mg, $59 \%$ yield) as a yellowish oil. $R_{\mathrm{f}} 0.39$ (EtOAc/cyclohexane 3:7). IR (neat): 3574, 3403, 3057, 2973, 2937, 1543, 1493, 1460, 1443, 1373, 1326, 1011, 909, 766, 731, $697 \mathrm{~cm}^{-1} .{ }^{1} \mathrm{H}$ NMR $\left(500 \mathrm{MHz}, \mathrm{CDCl}_{3}\right): \delta 7.41-7.37(\mathrm{~m}, 2 \mathrm{H}), 7.36-7.32$ $(\mathrm{m}, 2 \mathrm{H}), 7.31-7.26(\mathrm{~m}, 1 \mathrm{H}), 5.73(\mathrm{dd}, J=8.8,6.9 \mathrm{~Hz}, 1 \mathrm{H})$, 4.59-4.49 (m, 3H), 3.01 (dt, $J=15.0,8.8 \mathrm{~Hz}, 1 \mathrm{H}), 2.72$ (ddd, $J$ $=15.0,6.9,4.8 \mathrm{~Hz}, 1 \mathrm{H}), 2.07(\mathrm{ddq}, J=14.6,9.2,7.4 \mathrm{~Hz}, 1 \mathrm{H})$, $1.89(\mathrm{dqd}, J=14.6,7.4,4.7 \mathrm{~Hz}, 1 \mathrm{H}), 1.52(\mathrm{br} \mathrm{t}, J=5.8 \mathrm{~Hz}, 1 \mathrm{H})$, $1.01(\mathrm{t}, J=7.4 \mathrm{~Hz}, 3 \mathrm{H}) .{ }^{13} \mathrm{C} \mathrm{NMR}\left(126 \mathrm{MHz}, \mathrm{CDCl}_{3}\right): \delta \mathrm{C}$ 143.5, 140.4, 128.7, 127.9, 126.7, 124.5, 90.2, 59.9, 32.6, 27.1, 10.4; HRMS (ESI+/TOF) Calcd for $\mathrm{C}_{13} \mathrm{H}_{17} \mathrm{NO}_{3} \mathrm{Na}^{+}[\mathrm{M}+\mathrm{Na}]^{+}$: 258.1101. Found: 258.1106.

(Z)-5-Nitro-2-phenylnon-2-en-1-ol (3c). Compound 3c was prepared following the general procedure from cyclic carbonate 1 a $(190.2 \mathrm{mg}, 1.0 \mathrm{mmol})$ and 1-nitropentane. The product was purified by flash chromatography on silica gel using 10-20\% EtOAc/cyclohexane to afford the title compound $(212.2 \mathrm{mg}, 81$ $\%$ yield) as a dark red oil. $R_{\mathrm{f}} 0.31$ (EtOAc/cyclohexane $2: 8$ ). IR (neat): 3577, 3400, 3057, 2958, 2930, 2872, 1545, 1493, 1465, 1436, 1366, 1266, 1016, 764, 736, $697 \mathrm{~cm}^{-1} .{ }^{1} \mathrm{H}$ NMR $(500$ $\left.\mathrm{MHz}, \mathrm{CDCl}_{3}\right): \delta 7.41-7.37(\mathrm{~m}, 2 \mathrm{H}), 7.36-7.31(\mathrm{~m}, 2 \mathrm{H}), 7.31-$ $7.26(\mathrm{~m}, 1 \mathrm{H}), 5.73(\mathrm{dd}, J=8.5,6.9 \mathrm{~Hz}, 1 \mathrm{H}), 4.64-4.49(\mathrm{~m}, 3 \mathrm{H})$, 3.01 (ddd, $J=15.0,9.2,8.5 \mathrm{~Hz}, 1 \mathrm{H}), 2.71$ (ddd, $J=15.0,6.9$, $4.7 \mathrm{~Hz}, 1 \mathrm{H}), 2.11-2.01(\mathrm{~m}, 1 \mathrm{H}), 1.85-1.77$ (m, 1H), 1.49 (app $\mathrm{dd}, J=6.5,5.2 \mathrm{~Hz}, 1 \mathrm{H}), 1.41-1.30(\mathrm{~m}, 4 \mathrm{H}), 0.95-0.89(\mathrm{~m}, 3 \mathrm{H})$. ${ }^{13} \mathrm{C}$ NMR $\left(126 \mathrm{MHz}, \mathrm{CDCl}_{3}\right): \delta \mathrm{C} 143.5,140.4,128.7,127.9$, 126.7, 124.5, 88.8, 59.9, 33.5, 32.9, 28.0, 22.2, 13.9. HRMS (ESI+/TOF) Calcd for $\mathrm{C}_{15} \mathrm{H}_{21} \mathrm{NO}_{3} \mathrm{Na}^{+}[\mathrm{M}+\mathrm{Na}]^{+}: 286.1414$. Found: 286.1416. 
(Z)-5-Nitro-2,5-diphenylpent-2-en-1-ol (3d). Compound 3d was prepared following the general procedure from cyclic carbonate 1a $(190.2 \mathrm{mg}, \quad 1.0 \quad \mathrm{mmol})$ and (nitromethyl)benzene. The product was purified by flash chromatography on silica gel using 10-20\% EtOAc/cyclohexane to afford the title compound (209.4 mg, $74 \%$ yield) as a yellowish oil. $R_{\mathrm{f}} 0.26$ (EtOAc/cyclohexane 2:8). IR (neat): 3574, 3402, 3061, 3032, 2899, 1546, 1494, 1455, 1364, 1010, 909, 765, 717, $694 \mathrm{~cm}^{-1} .{ }^{1} \mathrm{H}$ NMR $\left(500 \mathrm{MHz}, \mathrm{CDCl}_{3}\right): \delta 7.54-7.49(\mathrm{~m}, 2 \mathrm{H})$, $7.46-7.41(\mathrm{~m}, 3 \mathrm{H}), 7.38-7.26(\mathrm{~m}, 5 \mathrm{H}), 5.72(\mathrm{dd}, J=8.2,6.9$ $\mathrm{Hz}, 1 \mathrm{H}), 5.60(\mathrm{dd}, J=9.1,6.0 \mathrm{~Hz}, 1 \mathrm{H}), 4.57(\mathrm{~d}, J=12.5 \mathrm{~Hz}$, $1 \mathrm{H}), 4.53(\mathrm{~d}, J=12.5 \mathrm{~Hz}, 1 \mathrm{H}), 3.53$ (ddd, $J=15.0,9.1,8.2 \mathrm{~Hz}$, 1H), 3.03 (ddd, $J=15.0,6.9,6.0 \mathrm{~Hz}, 1 \mathrm{H}), 1.60$ (br s, $1 \mathrm{H}) .{ }^{13} \mathrm{C}$ NMR (126 MHz, $\left.\mathrm{CDCl}_{3}\right): \delta 143.6,140.3,134.1,130.2,129.3$, 128.7, 127.9, 127.7, 126.6, 124.2, 91.1, 60.0, 33.3. HRMS (ESI+/TOF) Calcd for $\mathrm{C}_{17} \mathrm{H}_{17} \mathrm{NO}_{3} \mathrm{Na}^{+}[\mathrm{M}+\mathrm{Na}]^{+}$: 306.1101 . Found: 306.1110.

5-Nitro-2-phenylhex-2-ene-1,6-diol (3e). Compound 3e was prepared following the general procedure from cyclic carbonate 1a (190.2 mg, $1.0 \mathrm{mmol})$ and 2-nitroethanol. The product was purified by flash chromatography on silica gel using 30$35 \%$ EtOAc/cyclohexane to afford the title compound (106.5 $\mathrm{mg}, 45 \%$ yield) as a yellowish oil. $R_{\mathrm{f}} 0.16$ (EtOAc/cyclohexane 4:6). IR (neat): 3338, 3082, 3056, 3027, 2928, 2893, 2477, 2071, 1542, 1493, 1445, 1362, 1012, 972, 853, 766, $697 \mathrm{~cm}^{-1}$. ${ }^{1} \mathrm{H}$ NMR $\left(500 \mathrm{MHz}, \mathrm{CD}_{3} \mathrm{OD}\right): \delta 7.47-7.37(\mathrm{~m}, 2 \mathrm{H}), 7.31$ (ddt, $J=8.1,6.3,1.5 \mathrm{~Hz}, 2 \mathrm{H}), 7.27-7.21(\mathrm{~m}, 1 \mathrm{H}), 5.76(\mathrm{t}, J=7.7 \mathrm{~Hz}$, $1 \mathrm{H}), 4.76$ (tdd, $J=8.1,5.9,3.7 \mathrm{~Hz}, 1 \mathrm{H}), 4.49-4.42(\mathrm{~m}, 2 \mathrm{H})$, $4.00(\mathrm{dd}, J=12.2,7.8 \mathrm{~Hz}, 1 \mathrm{H}), 3.93(\mathrm{dd}, J=12.2,3.7 \mathrm{~Hz}, 1 \mathrm{H})$, $2.94(\mathrm{dt}, J=15.0,8.1 \mathrm{~Hz}, 1 \mathrm{H}), 2.82$ (ddd, $J=15.0,7.6,5.9 \mathrm{~Hz}$, $1 \mathrm{H}) .{ }^{13} \mathrm{C}$ NMR $\left(126 \mathrm{MHz}, \mathrm{CD}_{3} \mathrm{OD}\right): \delta 144.5,142.5,129.3$, $128.3,127.5,125.6,95.8,64.5,59.8,29.7$. Note: NMR resonances reported correspond to the $(Z)$ isomer. HRMS (ESI+/TOF) Calcd for $\mathrm{C}_{12} \mathrm{H}_{15} \mathrm{NO}_{4} \mathrm{Na}^{+}[\mathrm{M}+\mathrm{Na}]^{+}: 260.0893$. Found: 260.0896.

(Z)-7-Chloro-5-nitro-2-phenylhept-2-en-1-ol (3f). Compound $3 f$ was prepared following the general procedure from cyclic carbonate $\mathbf{1 a}(44.5 \mathrm{mg}, 0.23 \mathrm{mmol})$ and nitroalkane $\mathbf{2 h}$. The product was purified by flash chromatography on silica gel using 10-20\% EtOAc/cyclohexane to afford the title compound (38.0 mg, $60 \%$ yield) as a yellowish oil. $R_{\mathrm{f}} 0.15$ (EtOAc/cyclohexane 2:8). IR (neat): 3575, 3383, 3082, 3057, 3028, 2924, 2853, 1545, 1493, 1445, 1375, 1331, 1293, 1010, 971, 910, 767, 735, $697 \mathrm{~cm}^{-1} .{ }^{1} \mathrm{H}$ NMR $\left(400 \mathrm{MHz}, \mathrm{CDCl}_{3}\right): \delta 7.42-7.27(\mathrm{~m}$, $5 \mathrm{H}), 5.73(\mathrm{dd}, J=8.2,7.1 \mathrm{~Hz}, 1 \mathrm{H}), 4.91$ (tdd, $J=9.1,5.2,4.0$ $\mathrm{Hz}, 1 \mathrm{H}), 4.55(\mathrm{~d}, J=12.7 \mathrm{~Hz}, 1 \mathrm{H}), 4.50(\mathrm{~d}, J=12.7 \mathrm{~Hz}, 1 \mathrm{H})$, $3.65(\mathrm{dt}, J=11.3,5.5 \mathrm{~Hz}, 1 \mathrm{H}), 3.52(\mathrm{ddd}, J=11.6,9.1,4.9 \mathrm{~Hz}$, $1 \mathrm{H}), 3.02(\mathrm{dt}, J=15.0,8.4 \mathrm{~Hz}, 1 \mathrm{H}), 2.81(\mathrm{ddd}, J=15.0,7.1,5.2$ $\mathrm{Hz}, 1 \mathrm{H}), 2.58$ (ddt, $J=14.8,9.9,5.1 \mathrm{~Hz}, 1 \mathrm{H}$ ), 2.22 (dddd, $J=$ 15.0, 9.4, 5.7, 4.0 Hz, 1H), 1.67 (br s, $1 \mathrm{H}) .{ }^{13} \mathrm{C} \mathrm{NMR}(101 \mathrm{MHz}$, $\left.\mathrm{CDCl}_{3}\right): \delta 144.0,140.2,128.7,128.0,126.6,123.6,85.2,59.9$, 40.4, 35.7, 32.6. HRMS (ESI+/TOF) Calcd for $\mathrm{C}_{13} \mathrm{H}_{16} \mathrm{ClNO}_{3} \mathrm{Na}^{+}[\mathrm{M}+\mathrm{Na}]^{+}:$292.0711. Found: 292.0713 .

\section{(Z)-5-Nitro-2-phenyl-5-(phenylsulfonyl)pent-2-en-1-ol}

$(\mathbf{3 g})$. Compound $\mathbf{3 g}$ was prepared following the general procedure from cyclic carbonate $1 \mathrm{a}(190.2 \mathrm{mg}, 1.0 \mathrm{mmol})$ and nitromethyl phenyl sulfone. The product was purified by flash chromatography on silica gel using $20 \%$ EtOAc/cyclohexane to afford the title compound (249.2 mg, $72 \%$ yield) as a yellowish oil. $R_{\mathrm{f}} 0.22$ (EtOAc/cyclohexane 3:7). IR (neat): 3563, 3064, 2963, 1558, 1493, 1448, 1334, 1221, 1154, 1082, 999, 908, 757, $724,684,600,574,530 \mathrm{~cm}^{-1} .{ }^{1} \mathrm{H} \mathrm{NMR}\left(400 \mathrm{MHz} \mathrm{CDCl}_{3}\right): \delta \mathrm{H}$
7.96-7.89 (m, 2H), 7.81-7.74 (m, 1H), 7.67-7.59 (m, 2H), $7.36-7.26(\mathrm{~m}, 5 \mathrm{H}), 5.71(\mathrm{dd}, J=9.2,5.3 \mathrm{~Hz}, 1 \mathrm{H}), 5.65(\mathrm{t}, J=$ $7.7 \mathrm{~Hz}, 1 \mathrm{H}), 4.55(\mathrm{~d}, J=12.7 \mathrm{~Hz}, 1 \mathrm{H}), 4.51(\mathrm{~d}, J=12.7 \mathrm{~Hz}$, $1 \mathrm{H}), 3.38-3.24(\mathrm{~m}, 2 \mathrm{H}), 1.88$ (br s, 1H). ${ }^{13} \mathrm{C}$ NMR (101 MHz, $\left.\mathrm{CDCl}_{3}\right): \delta 145.6,139.9,135.8,134.1,130.0,129.8,128.7$, 128.2, 126.6, 120.8, 101.6, 60.2, 27.3. HRMS (ESI+/TOF) Calcd for $\mathrm{C}_{17} \mathrm{H}_{17} \mathrm{NO}_{5} \mathrm{SNa}^{+}\left[\mathrm{M}+\mathrm{Na}^{+}\right.$: 370.0720. Found: 370.0729 .

(Z)-Ethyl-6-hydroxy-2-nitro-5-phenylhex-4-enoate (3h). Compound $\mathbf{3 h}$ was prepared following the general procedure from cyclic carbonate 1a $(190.2 \mathrm{mg}, 1.0 \mathrm{mmol})$ and ethyl nitroacetate. The product was purified by flash chromatography on silica gel using $20 \%$ EtOAc/cyclohexane to afford the title compound (223.0 mg, $80 \%$ yield) as a yellowish oil. $R_{\mathrm{f}} 0.30$ (EtOAc/cyclohexane 3:7). IR (neat): 3576, 2984, 1745, 1557, 1493, 1445, 1371, 1260, 1209, 1013, 857, 766, $698 \mathrm{~cm}^{-1} .{ }^{1} \mathrm{H}$ NMR (400 MHz, $\left.\mathrm{CDCl}_{3}\right) \delta 7.43-7.27(\mathrm{~m}, 5 \mathrm{H}), 5.73$ (app t, $J$ $=7.7 \mathrm{~Hz}, 1 \mathrm{H}), 5.26(\mathrm{dd}, J=8.6,6.0 \mathrm{~Hz}, 1 \mathrm{H}), 4.60(\mathrm{~d}, J=12.6$ $\mathrm{Hz}, 1 \mathrm{H}), 4.56(\mathrm{~d}, J=12.6 \mathrm{~Hz}, 1 \mathrm{H}), 4.31(\mathrm{qd}, J=7.2,1.8 \mathrm{~Hz}$, 2H), 3.29 (dt, $J=15.1,8.3 \mathrm{~Hz}, 1 \mathrm{H}$ ), 3.17 (ddd, $J=15.1,7.4,6.0$ $\mathrm{Hz}, 1 \mathrm{H}), 1.81$ (br s, 1H), 1.31 (t, $J=7.2 \mathrm{~Hz}, 3 \mathrm{H}) .{ }^{13} \mathrm{C}$ NMR $(101$ $\left.\mathrm{MHz}, \mathrm{CDCl}_{3}\right): \delta 164.2,144.5,140.2,128.7,128.1,126.6$, 122.7, 87.6, 63.5, 60.0, 29.7, 14.0. HRMS (ESI+/TOF) Calcd for $\mathrm{C}_{14} \mathrm{H}_{17} \mathrm{NO}_{5} \mathrm{Na}[\mathrm{M}+\mathrm{Na}]^{+}:$302.0999. Found: 302.0996.

(Z)-6-(1H-Indol-3-yl)-5-nitro-2-phenylhex-2-en-1-ol (3i). Compound $3 \mathbf{i}$ was prepared following the general procedure from cyclic carbonate $1 \mathrm{a}(111.1 \mathrm{mg}, 0.58 \mathrm{mmol})$ and nitroalkane $\mathbf{2 a}$. The product was purified by flash chromatography on silica gel using 20-30 \% EtOAc/cyclohexane to afford the title compound (126.7 mg, $64 \%$ yield) as a yellowish oil. $R_{\mathrm{f}} 0.08$ (EtOAc/cyclohexane 2:8). IR (neat): 3556, 3415, 3056, 2924, 1747, 1543, 1492, 1457, 1370, 1339, 1279, 1230, 1097, 1010, $743,697 \mathrm{~cm}^{-1} .{ }^{1} \mathrm{H}$ NMR (400 MHz, $\mathrm{CDCl}_{3}$ ): $\delta 8.15$ (br s, $1 \mathrm{H}$ ), $7.59(\mathrm{dt}, J=7.9,1.0 \mathrm{~Hz}, 1 \mathrm{H}), 7.41-7.27(\mathrm{~m}, 6 \mathrm{H}), 7.23$ (ddd, $J$ $=8.1,7.0,1.2 \mathrm{~Hz}, 1 \mathrm{H}), 7.17(\mathrm{ddd}, J=8.0,7.0,1.1 \mathrm{~Hz}, 1 \mathrm{H}), 7.04$ $(\mathrm{d}, J=2.5 \mathrm{~Hz}, 1 \mathrm{H}), 5.75(\mathrm{dd}, J=8.6,6.7 \mathrm{~Hz}, 1 \mathrm{H}), 4.96$ (dddd, $J=9.2,7.7,6.5,4.5 \mathrm{~Hz}, 1 \mathrm{H}), 4.53(\mathrm{~d}, J=12.5 \mathrm{~Hz}, 1 \mathrm{H}), 4.48(\mathrm{~d}$, $J=12.5 \mathrm{~Hz}, 1 \mathrm{H}), 3.54$ (ddd, $J=14.8,7.7,0.8 \mathrm{~Hz}, 1 \mathrm{H}), 3.32$ (ddd, $J=14.8,6.5,0.7 \mathrm{~Hz}, 1 \mathrm{H}), 3.08$ (dt, $J=15.2,8.9 \mathrm{~Hz}, 1 \mathrm{H})$, 2.80 (ddd, $J=15.2,6.8,4.5 \mathrm{~Hz}, 1 \mathrm{H}), 1.51$ (br s, $1 \mathrm{H}) .{ }^{13} \mathrm{C} \mathrm{NMR}$ $\left(101 \mathrm{MHz}, \mathrm{CDCl}_{3}\right): \delta 143.5,140.3,136.3,128.7,127.9,126.9$, 126.6, 124.5, 123.2, 122.7, 120.1, 118.3, 111.6, 109.6, 88.8, 59.9, 32.5, 30.0. HRMS (ESI+/TOF) Calcd for $\mathrm{C}_{20} \mathrm{H}_{20} \mathrm{~N}_{2} \mathrm{O}_{3} \mathrm{Na}^{+}$ $[\mathrm{M}+\mathrm{Na}]^{+}:$359.1366. Found: 359.1367 .

(Z)-5-Nitro-2-phenyl-6-(phenylamino)hex-2-en-1-ol (3j). Compound $\mathbf{3 j}$ was prepared following the general procedure from cyclic carbonate 1a $(190.2 \mathrm{mg}, 1.0 \mathrm{mmol})$ and nitroalkane 2d. The product was purified by flash chromatography on silica gel using 10-20\% EtOAc/cyclohexane to afford the title compound (150.6 mg, $48 \%$ yield) as a yellowish oil. $R_{\mathrm{f}} 0.18$ (EtOAc/cyclohexane 2:8). IR (neat): 3567, 3408, 3055, 3026, 2922, 1602, 1544, 1497, 1444, 1381, 1355, 1314, 1256, 1182, 992, 908, 750, $692 \mathrm{~cm}^{-1} .{ }^{1} \mathrm{H}$ NMR (400 MHz, $\left.\mathrm{CDCl}_{3}\right): \delta 7.42-$ $7.27(\mathrm{~m}, 6 \mathrm{H}), 7.24-7.17(\mathrm{~m}, 2 \mathrm{H}), 6.78(\mathrm{tt}, J=7.4,1.1 \mathrm{~Hz}, 1 \mathrm{H})$, $6.66-6.59$ (m, 2H), 5.77 (dd, $J=8.2,7.4 \mathrm{~Hz}, 1 \mathrm{H}), 4.88$ (tdd, $J$ $=8.0,5.6,4.5 \mathrm{~Hz}, 1 \mathrm{H}), 4.56(\mathrm{~s}, 2 \mathrm{H}), 4.13(\mathrm{br} \mathrm{s}, 1 \mathrm{H}), 3.81(\mathrm{dd}, J$ $=14.7,7.9 \mathrm{~Hz}, 1 \mathrm{H}), 3.65(\mathrm{dd}, J=14.8,4.5 \mathrm{~Hz}, 1 \mathrm{H}), 3.06(\mathrm{dt}, J$ $=15.0,8.1 \mathrm{~Hz}, 1 \mathrm{H}), 2.90(\mathrm{ddd}, J=15.0,7.4,5.6 \mathrm{~Hz}, 1 \mathrm{H}), 1.66$ (br s, $1 \mathrm{H}) .{ }^{13} \mathrm{C} \mathrm{NMR}\left(101 \mathrm{MHz}, \mathrm{CDCl}_{3}\right): \delta 146.4,143.9,140.3$, 129.7, 128.8, 128.0, 126.6, 123.8, 118.9, 113.2, 86.5, 60.0, 46.4, 30.4. HRMS (ESI+/TOF) Calcd for $\mathrm{C}_{18} \mathrm{H}_{21} \mathrm{~N}_{2} \mathrm{O}_{3}[\mathrm{M}+\mathrm{H}]^{+}$: 313.1547. Found: 313.1545. 
(Z)-tert-Butyl-8-hydroxy-4-nitro-7-phenyloct-6-enoate $(\mathbf{3 k})$. Compound $\mathbf{3 k}$ was prepared following the general procedure from cyclic carbonate $1 \mathrm{a}(190.2 \mathrm{mg}, 1.0 \mathrm{mmol})$ and nitroalkane $\mathbf{2 b}$. The product was purified by flash chromatography on silica gel using 10-20\% EtOAc/cyclohexane to afford the title compound $\left(215.7 \mathrm{mg}, 64 \%\right.$ yield) as a yellowish oil. $R_{\mathrm{f}}$ 0.17 (EtOAc/cyclohexane 2:8). IR (neat): 3566, 3434, 3083, 3059, 2979, 2933, 1724, 1547, 1493, 1445, 1367, 1320, 1251, 1150, 1009, 911, 844, 766, 732, $698 \mathrm{~cm}^{-1} .{ }^{1} \mathrm{H}$ NMR $(400 \mathrm{MHz}$, $\left.\mathrm{CDCl}_{3}\right): \delta 7.41-7.25(\mathrm{~m}, 5 \mathrm{H}), 5.72(\mathrm{dd}, J=8.3,7.1 \mathrm{~Hz}, 1 \mathrm{H})$, $4.74-4.64(\mathrm{~m}, 1 \mathrm{H}), 4.57-4.46(\mathrm{~m}, 2 \mathrm{H}), 2.99(\mathrm{dt}, J=15.0,8.6$ $\mathrm{Hz}, 1 \mathrm{H}), 2.74$ (ddd, $J=15.0,7.1,5.0 \mathrm{~Hz}, 1 \mathrm{H}), 2.40-2.08(\mathrm{~m}$, $4 \mathrm{H}), 1.88$ (br t, $J=5.1 \mathrm{~Hz}, 1 \mathrm{H}), 1.45$ (s, 9H). ${ }^{13} \mathrm{C}$ NMR (101 $\left.\mathrm{MHz}, \mathrm{CDCl}_{3}\right): \delta 171.3,143.7,140.4,128.6,127.8,126.6$, 124.0, 87.6, 81.4, 59.8, 32.8, 31.4, 28.6, 28.1. HRMS (ESI+/TOF) Calcd for $\mathrm{C}_{18} \mathrm{H}_{25} \mathrm{NO}_{5} \mathrm{Na}^{+}[\mathrm{M}+\mathrm{Na}]^{+}: 358.1625$. Found: 358.1633.

(Z)-6-(Furan-2-yl)-5-nitro-2-phenylhex-2-en-1-ol $(3 l)$. Compound 3I was prepared following the general procedure from cyclic carbonate 1a $(190.2 \mathrm{mg}, 1.0 \mathrm{mmol})$ and nitroalkane 2c. The product was purified by flash chromatography on silica gel using 10-20\% EtOAc/cyclohexane to afford the title compound $\left(218.3 \mathrm{mg}, 76 \%\right.$ yield) as a yellowish oil. $R_{\mathrm{f}} 0.22$ (EtOAc/cyclohexane 2:8). IR (neat): 3574, 3398, 3027, 2920, 1599, 1547, 1506, 1493, 1429, 1371, 1325, 1208, 1145, 1077, $1010,911,732,697 \mathrm{~cm}^{-1} .{ }^{1} \mathrm{H}$ NMR $\left(400 \mathrm{MHz}, \mathrm{CDCl}_{3}\right): \delta 7.42$ $7.26(\mathrm{~m}, 6 \mathrm{H}), 6.31(\mathrm{dd}, J=3.2,1.9 \mathrm{~Hz}, 1 \mathrm{H}), 6.19-6.13(\mathrm{~m}, 1 \mathrm{H})$, 5.73 (dd, $J=8.4,7.0 \mathrm{~Hz}, 1 \mathrm{H}), 4.91$ (dddd, $J=8.8,7.9,6.0,4.9$ $\mathrm{Hz}, 1 \mathrm{H}), 4.54-4.45(\mathrm{~m}, 2 \mathrm{H}), 3.40(\mathrm{dd}, J=15.4,7.9 \mathrm{~Hz}, 1 \mathrm{H})$, $3.20(\mathrm{dd}, J=15.4,6.0 \mathrm{~Hz}, 1 \mathrm{H}), 3.01(\mathrm{dt}, J=15.2,8.6 \mathrm{~Hz}, 1 \mathrm{H})$, 2.79 (ddd, $J=15.2,7.1,4.9 \mathrm{~Hz}, 1 \mathrm{H}$ ), 1.80 (br s, $1 \mathrm{H}) .{ }^{13} \mathrm{C} \mathrm{NMR}$ $\left(101 \mathrm{MHz}, \mathrm{CDCl}_{3}\right): \delta 149.1,143.7,142.5,140.3,128.6,127.9$, $126.6,123.8,110.7,108.3,86.6,59.7,32.1,31.9$. HRMS (ESI+/TOF) Calcd for $\mathrm{C}_{16} \mathrm{H}_{17} \mathrm{NO}_{4} \mathrm{Na}^{+}[\mathrm{M}+\mathrm{Na}]^{+}: 310.1050$. Found: 310.1046.

(Z)-7,7-Dimethoxy-5-nitro-2-phenylhept-2-en-1-ol (3m). Compound $\mathbf{3 m}$ was prepared following the general procedure from cyclic carbonate 1a $(190.2 \mathrm{mg}, 1.0 \mathrm{mmol})$ and nitroalkane 2e. The product was purified by flash chromatography on silica gel using 20-30 \% EtOAc/cyclohexane to afford the title compound $\left(281.0 \mathrm{mg}, 95 \%\right.$ yield) as a yellowish oil. $R_{\mathrm{f}} 0.08$ (EtOAc/cyclohexane 2:8). IR (neat): 3568, 3422, 3081, 3058, 3029, 2992, 2937, 2911, 2836, 2251, 1548, 1493, 1445, 1370, 1325, 1267, 1194, 1127, 1065, 1023, 949, 909, 768, 730, 698 $\mathrm{cm}^{-1} .{ }^{1} \mathrm{H}$ NMR $\left(500 \mathrm{MHz}, \mathrm{CDCl}_{3}\right): \delta 7.42-7.37(\mathrm{~m}, 2 \mathrm{H}), 7.37$ $7.32(\mathrm{~m}, 2 \mathrm{H}), 7.31-7.27(\mathrm{~m}, 1 \mathrm{H}), 5.73(\mathrm{dd}, J=8.3,7.1 \mathrm{~Hz}, 1 \mathrm{H})$, 4.77 (tdd, $J=9.1,5.1,4.2 \mathrm{~Hz}, 1 \mathrm{H}), 4.58-4.47$ (m, 2H), 4.41 (dd, $J=6.3,4.5 \mathrm{~Hz}, 1 \mathrm{H}), 3.37$ (s, 3H), 3.35 (s, 3H), 2.99 (dt, $J=$ 14.9, 8.5 Hz, 1H), 2.79 (ddd, $J=15.0,7.1,5.1 \mathrm{~Hz}, 1 \mathrm{H}), 2.45$ (ddd, $J=14.7,9.2,4.5 \mathrm{~Hz}, 1 \mathrm{H}$ ), 2.05 (ddd, $J=14.7,6.3,4.2$ $\mathrm{Hz}, 1 \mathrm{H}$ ), 1.53 (br t, $J=4.7 \mathrm{~Hz}, 1 \mathrm{H}) .{ }^{13} \mathrm{C} \mathrm{NMR}(126 \mathrm{MHz}$, $\left.\mathrm{CDCl}_{3}\right): \delta 143.8,140.3,128.7,128.0,126.7,124.0,102.1,84.5$, 59.9, 54.5, 54.1, 36.4, 33.1. HRMS (ESI+/TOF) Calcd for $\mathrm{C}_{15} \mathrm{H}_{21} \mathrm{NO}_{5} \mathrm{Na}[\mathrm{M}+\mathrm{Na}]^{+}:$318.1312. Found: 318.1321 .

(Z)-6,6-Bis(methylthio)-5-nitro-2-phenylhex-2-en-1-ol $(3 n)$. Compound $3 n$ was prepared following the general procedure from cyclic carbonate $1 \mathrm{a}(190.2 \mathrm{mg}, 1.0 \mathrm{mmol})$ and nitroalkane $\mathbf{2 g}$. The product was purified by flash chromatography on silica gel using $10 \%$ EtOAc/cyclohexane to afford the title compound (170.3 mg, $54 \%$ yield) as a yellowish oil. $R_{\mathrm{f}}$ 0.22 (EtOAc/cyclohexane 2:8). IR (neat): 3565, 3396, 3081, 3055, 3026, 2919, 2854, 1550, 1493, 1423, 1365, 1308, 1011,
975, 957, 766, $698 \mathrm{~cm}^{-1} .{ }^{1} \mathrm{H}$ NMR (500 MHz, $\left.\mathrm{CDCl}_{3}\right): \delta 7.41-$ 7.37 (m, 2H), 7.37-7.31 (m, 2H), 7.31-7.27 (m, 1H), 5.76 (dd, $J=8.7,6.7 \mathrm{~Hz}, 1 \mathrm{H}), 4.70(\mathrm{td}, J=9.7,3.7 \mathrm{~Hz}, 1 \mathrm{H}), 4.57(\mathrm{dd}, J$ $=12.7,3.4 \mathrm{~Hz}, 1 \mathrm{H}), 4.53(\mathrm{dd}, J=12.7,5.2 \mathrm{~Hz}, 1 \mathrm{H}), 4.13(\mathrm{~d}, J$ $=9.6 \mathrm{~Hz}, 1 \mathrm{H}), 3.19(\mathrm{ddd}, J=15.3,6.7,3.7 \mathrm{~Hz}, 1 \mathrm{H}), 3.09($ app $\mathrm{dt}, J=15.3,9.3 \mathrm{~Hz}, 1 \mathrm{H}$ ), 2.21 (s, 3H), 2.19 (s, 3H), 1.57 (overlapped, $1 \mathrm{H}) .{ }^{13} \mathrm{C} \mathrm{NMR}\left(126 \mathrm{MHz}, \mathrm{CDCl}_{3}\right): \delta 144.1,140.3$, 128.7, 128.0, 126.7, 123.8, 90.7, 60.0, 55.7, 31.5, 13.7, 13.5; HRMS (ESI+/TOF) Calcd for $\mathrm{C}_{14} \mathrm{H}_{19} \mathrm{NO}_{3} \mathrm{~S}_{2} \mathrm{Na}^{+}[\mathrm{M}+\mathrm{Na}]^{+}$: 336.0699. Found: 336.0700.

(Z)-2-(6-Hydroxy-2-nitro-5-phenylhex-4-en-1-yl)isoindoline-1,3-dione (3o). Compound 30 was prepared following the general procedure from cyclic carbonate 1a (190.2 mg, 1.0 $\mathrm{mmol}$ ) and nitroalkane $\mathbf{2 f}$. The product was purified by flash chromatography on silica gel using 20-30 \% EtOAc/cyclohexane to afford the title compound (251.1 mg, $69 \%$ yield) as a pale yellow solid. $R_{\mathrm{f}} 0.11$ (EtOAc/cyclohexane 3:7). IR (neat): 3455, 3090, 3060, 3029, 2954, 2897, 1774, 1702, 1611, 1552, 1473, 1423, 1396, 1339, 1296, 1256, 1192, 1088, 1017, 992, $969,931,904,881,851,771,715,690 \mathrm{~cm}^{-1} .{ }^{1} \mathrm{H}$ NMR $(400$ $\left.\mathrm{MHz}, \mathrm{CDCl}_{3}\right): \delta 7.92-7.81(\mathrm{~m}, 2 \mathrm{H}), 7.79-7.71(\mathrm{~m}, 2 \mathrm{H}), 7.40$ $7.27(\mathrm{~m}, 5 \mathrm{H}), 5.75(\mathrm{t}, J=7.6 \mathrm{~Hz}, 1 \mathrm{H}), 5.06(\mathrm{tt}, J=8.4,5.1 \mathrm{~Hz}$, $1 \mathrm{H}), 4.57(\mathrm{~d}, J=4.8 \mathrm{~Hz}, 2 \mathrm{H}), 4.42(\mathrm{dd}, J=14.5,8.3 \mathrm{~Hz}, 1 \mathrm{H})$, $4.06(\mathrm{dd}, J=14.5,4.8 \mathrm{~Hz}, 1 \mathrm{H}), 3.11(\mathrm{dt}, J=15.2,8.3 \mathrm{~Hz}, 1 \mathrm{H})$, 2.90 (ddd, $J=15.2,7.2,5.3 \mathrm{~Hz}, 1 \mathrm{H}), 1.66$ (br t, $J=5.8 \mathrm{~Hz}, 1 \mathrm{H})$. ${ }^{13} \mathrm{C}$ NMR $\left(101 \mathrm{MHz}, \mathrm{CDCl}_{3}\right): \delta 167.7,144.3,140.1,134.7$, 131.7, 128.7, 128.0, 126.6, 123.9, 122.9, 84.9, 60.0, 40.0, 30.6. HRMS (ESI+/TOF) Calcd for $\mathrm{C}_{20} \mathrm{H}_{18} \mathrm{~N}_{2} \mathrm{O}_{5} \mathrm{Na}^{+}[\mathrm{M}+\mathrm{Na}]^{+}$: 389.1108. Found: 389.1111.

(Z)-5-Nitro-2-phenylpent-2-en-1-yl benzoate (3q). Compound $3 q$ was prepared following the general procedure from cyclic carbonate $\mathbf{1 a}(38.0 \mathrm{mg}, 0.20 \mathrm{mmol})$ and benzoyl nitromethane. The product was purified by flash chromatography on silica gel using $5 \%$ EtOAc/cyclohexane to afford the title compound (41.9 mg, $67 \%$ yield) as a brownish oil. $R_{\mathrm{f}} 0.26$ (EtOAc/cyclohexane 1:9). IR (neat): 3061, 3031, 2962, 2918, 1807, 1713, 1600, 1548, 1493, 1450, 1377, 1315, 1265, 1176, 1106, 1069, 1025, 954, 763, $710 \mathrm{~cm}^{-1} .{ }^{1} \mathrm{H}$ NMR (400 MHz, $\left.\mathrm{CDCl}_{3}\right): \delta 7.98-7.91(\mathrm{~m}, 2 \mathrm{H}), 7.54(\mathrm{app} \mathrm{dt}, J=8.8,1.4 \mathrm{~Hz}, 1 \mathrm{H})$, $7.45-7.27(\mathrm{~m}, 7 \mathrm{H}), 5.96(\mathrm{t}, J=7.6 \mathrm{~Hz}, 1 \mathrm{H}), 5.28(\mathrm{~s}, 2 \mathrm{H}), 4.55$ $(\mathrm{t}, J=6.8 \mathrm{~Hz}, 2 \mathrm{H}), 3.12(\mathrm{q}, J=7.0 \mathrm{~Hz}, 2 \mathrm{H}) .{ }^{13} \mathrm{C}$ NMR $(101$ $\left.\mathrm{MHz}, \mathrm{CDCl}_{3}\right): \delta 166.5,140.0,138.9,133.3,130.0,129.8$, 128.7, 128.6, 128.0, 127.3, 126.5, 74.9, 61.6, 26.8. HRMS (ESI+/TOF) Calcd for $\mathrm{C}_{18} \mathrm{H}_{17} \mathrm{NO}_{4} \mathrm{Na}^{+}[\mathrm{M}+\mathrm{Na}]^{+}: 334.1050$. Found: 334.1054.

2-Methyl-5-nitrohex-2-en-1-ol (4a). Compound 4a was prepared following the general procedure from 4-methyl-4-vinyl1,3-dioxolan-2-one (128.1 mg, $1.0 \mathrm{mmol}$ ) and nitroethane. The product was purified by flash chromatography on silica gel using 20-30\% EtOAc/cyclohexane to afford the title compound (90.0 mg, $57 \%$ yield) as a yellowish oil. $R_{\mathrm{f}} 0.31$ (EtOAc/cyclohexane 3:7). IR (neat): 3377, 2976, 2941, 1542, 1451, 1388, $1361,1008 \mathrm{~cm}^{-1} .{ }^{1} \mathrm{H}$ NMR $\left(400 \mathrm{MHz}, \mathrm{CDCl}_{3}\right): \delta 5.25-5.17(\mathrm{~m}$, $1 \mathrm{H}), 4.62-4.51(\mathrm{~m}, 1 \mathrm{H}), 4.13$ (app d, $J=12.3 \mathrm{~Hz}, 1 \mathrm{H}), 4.06$ (dd, $J=12.6,3.4 \mathrm{~Hz}, 1 \mathrm{H}), 2.85-2.70(\mathrm{~m}, 1 \mathrm{H}), 2.55-2.42(\mathrm{~m}, 1 \mathrm{H})$, $1.80(\mathrm{q}, J=1.2 \mathrm{~Hz}, 3 \mathrm{H}), 1.53(\mathrm{~d}, J=6.7 \mathrm{~Hz}, 3 \mathrm{H}), 1.51$ (overlapped, $1 \mathrm{H}) .{ }^{13} \mathrm{C} \mathrm{NMR}\left(101 \mathrm{MHz}, \mathrm{CDCl}_{3}\right): \delta 139.9,120.6,83.6$, 61.4, 33.4, 21.6, 18.9. Note: the NMR resonances reported correspond to the $(Z)$ isomer. HRMS (ESI+/TOF) Calcd for $\mathrm{C}_{7} \mathrm{H}_{13} \mathrm{NO}_{3} \mathrm{Na}^{+}[\mathrm{M}+\mathrm{Na}]^{+}:$182.0788. Found: 182.0787.

5-Nitrohex-2-en-1-ol (4b). Compound $4 \mathbf{b}$ was prepared following the general procedure from 4-vinyl-1,3-dioxolan-2-one 
(114.1 $\mathrm{mg}, 1.0 \mathrm{mmol}$ ) and nitroethane. The product was purified by flash chromatography on silica gel using 20-30\% EtOAc/cyclohexane to afford the title compound $(96.1 \mathrm{mg}, 66$ $\%$ yield) as a yellowish oil. $R_{\mathrm{f}} 0.16$ (EtOAc/cyclohexane $3: 7$ ). IR (neat): 3570, 3353, 2940, 1543, 1451, 1389, 1361, 1314, $1004,973,857 \mathrm{~cm}^{-1} .{ }^{1} \mathrm{H} \mathrm{NMR}\left(500 \mathrm{MHz}, \mathrm{CDCl}_{3}\right): \delta 5.78$ (overlapped, $1 \mathrm{H}), 5.46$ (dddt, $J=11.1,8.5,7.2,1.5 \mathrm{~Hz}, 1 \mathrm{H}), 4.63-$ $4.55(\mathrm{~m}, 1 \mathrm{H}), 4.25-4.14(\mathrm{~m}, 2 \mathrm{H}), 2.86-2.77(\mathrm{~m}, 1 \mathrm{H}), 2.52$ (overlapped, 1H), 1.56 (dd, $J=12.1,6.7 \mathrm{~Hz}, 3 \mathrm{H}), 1.45$ (br s, 1H). $\left.{ }^{13} \mathrm{C} \mathrm{NMR} \mathrm{(126} \mathrm{MHz,} \mathrm{CDCl}_{3}\right): \delta 133.4,125.1,83.0,58.4$, 33.1, 18.9. Note: NMR resonances reported correspond to the (Z) isomer. HRMS (ESI+/TOF) Calcd for $\mathrm{C}_{6} \mathrm{H}_{11} \mathrm{NO}_{3} \mathrm{Na}^{+}[\mathrm{M}+$ $\mathrm{Na}^{+}:$168.0631. Found: 168.0632.

(Z)-2-Cyclohexyl-5-nitrohex-2-en-1-ol (4c). Compound 4c was prepared following the general procedure from 4-cyclohexyl-4-vinyl-1,3-dioxolan-2-one (39.3 mg, $0.20 \mathrm{mmol}$ ) and nitroethane. The product was purified by flash chromatography on silica gel using $20 \% \mathrm{EtOAc/cyclohexane} \mathrm{to} \mathrm{afford} \mathrm{the} \mathrm{title}$ compound (32.0 mg, $70 \%$ yield) as a brownish oil. $R_{\mathrm{f}} 0.42$ (EtOAc/cyclohexane 3:7). IR (neat): 3287, 3221, 2923, 2851, $1545,1448,1387,1360,998 \mathrm{~cm}^{-1} .{ }^{1} \mathrm{H} \mathrm{NMR}\left(400 \mathrm{MHz}, \mathrm{CDCl}_{3}\right)$ : $\delta 5.23(\mathrm{ddd}, J=8.1,6.9,1.0 \mathrm{~Hz}, 1 \mathrm{H}), 4.60(\mathrm{dqd}, J=8.3,6.7$, $5.4 \mathrm{~Hz}, 1 \mathrm{H}), 4.14$ (dd, $J=11.9,4.6 \mathrm{~Hz}, 1 \mathrm{H}), 4.09$ (dd, $J=11.9$, $5.7 \mathrm{~Hz}, 1 \mathrm{H}), 2.83(\mathrm{dt}, J=14.7,8.3 \mathrm{~Hz}, 1 \mathrm{H}), 2.55(\mathrm{dt}, J=14.7$, 6.7, $5.7 \mathrm{~Hz}, 1 \mathrm{H}), 2.09-1.98(\mathrm{~m}, 1 \mathrm{H}), 1.83-1.63(\mathrm{~m}, 6 \mathrm{H}), 1.55$ $(\mathrm{d}, J=6.7 \mathrm{~Hz}, 3 \mathrm{H}), 1.35-1.05(\mathrm{~m}, 5 \mathrm{H}) .{ }^{13} \mathrm{C} \mathrm{NMR}(101 \mathrm{MHz}$, $\left.\mathrm{CDCl}_{3}\right): \delta 149.2,119.8,83.8,59.7,43.6,33.5,32.7,32.6,26.82$, 26.79, 26.3, 19.0. HRMS (ESI+/TOF) Calcd for $\mathrm{C}_{12} \mathrm{H}_{21} \mathrm{NO}_{3} \mathrm{Na}^{+}$ $[\mathrm{M}+\mathrm{Na}]^{+}: 250.1414$. Found: 250.1412 .

(Z)-2-([1,1'-Biphenyl]-4-yl)-5-nitrohex-2-en-1-ol (4d). Compound 4d was prepared following the general procedure from 4-([1,1'-biphenyl]-4-yl)-4-vinyl-1,3-dioxolan-2-one (57.6 $\mathrm{mg}, 0.22 \mathrm{mmol})$ and nitroethane. The product was purified by flash chromatography on silica gel using 20-30\% EtOAc/cyclohexane to afford the title compound (34.6 mg, $54 \%$ yield) as a yellowish oil. $R_{\mathrm{f}} 0.30$ (EtOAc/cyclohexane $\left.3: 7\right)$. IR (neat): 3428, 3029, 2924, 1538, 1486, 1448, 1388, 1357, 1000, 837, 763, 730, $692 \mathrm{~cm}^{-1} .{ }^{1} \mathrm{H}$ NMR $\left(500 \mathrm{MHz}, \mathrm{CDCl}_{3}\right): \delta 7.62-7.56$ (m, 4H), 7.51-7.47 (m, 2H), 7.47-.42 (m, 2H), 7.38-7.33 (m, $1 \mathrm{H}), 5.81(\mathrm{dd}, J=8.3,7.2 \mathrm{~Hz}, 1 \mathrm{H}), 4.73(\mathrm{dqd}, J=8.1,6.6,5.3$ $\mathrm{Hz}, 1 \mathrm{H}), 4.62(\mathrm{~d}, J=12.5 \mathrm{~Hz}, 1 \mathrm{H}), 4.58(\mathrm{~d}, J=12.5 \mathrm{~Hz}, 1 \mathrm{H})$, $3.05(\mathrm{dt}, J=14.9,8.3 \mathrm{~Hz}, 1 \mathrm{H}), 2.77(\mathrm{ddd}, J=14.9,7.2,5.4 \mathrm{~Hz}$, $1 \mathrm{H}), 1.64$ (d, $J=6.7 \mathrm{~Hz}, 3 \mathrm{H}), 1.56$ (br s, $1 \mathrm{H}) .{ }^{13} \mathrm{C}$ NMR $(126$ $\left.\mathrm{MHz}, \mathrm{CDCl}_{3}\right): \delta 143.1,140.8,140.7,139.2,128.9,127.5$, 127.4, 127.1, 127.0, 124.3, 83.3, 59.8, 34.1, 19.1. HRMS (ESI+/TOF) Calcd for $\mathrm{C}_{18} \mathrm{H}_{19} \mathrm{NO}_{3} \mathrm{Na}^{+}[\mathrm{M}+\mathrm{Na}]^{+}: 320.1257$. Found: 320.1271.

(Z)-5-Nitro-2-(3-(trifluoromethyl)phenyl)hex-2-en-1-ol $(4 \mathrm{e})$. Compound $4 \mathrm{e}$ was prepared following the general procedure from cyclic carbonate $1 \mathrm{c}(258.2 \mathrm{mg}, 1.0 \mathrm{mmol})$ and nitroethane. The product was purified by flash chromatography on silica gel using 20-30\% EtOAc/cyclohexane to afford the title compound (100.0 mg, $35 \%$ yield) as a yellowish oil. $R_{\mathrm{f}}$ 0.19 (EtOAc/cyclohexane 3:7). IR (neat): 3578, 3371, 3077, 2992, 2924, 2899, 2854, 1806, 1547, 1489, 1436, 1390, 1331, 1262, 1164, 1118, 1075, 1066, 907, 800, 733, $701 \mathrm{~cm}^{-1} .{ }^{1} \mathrm{H}$ NMR (400 MHz, $\left.\mathrm{CDCl}_{3}\right): \delta 7.64(\mathrm{~d}, J=1.8 \mathrm{~Hz}, 1 \mathrm{H}), 7.62-7.52$ $(\mathrm{m}, 2 \mathrm{H}), 7.45(\mathrm{~d}, J=7.7 \mathrm{~Hz}, 1 \mathrm{H}), 5.80(\mathrm{dd}, J=8.4,7.0 \mathrm{~Hz}, 1 \mathrm{H})$, $4.73(\mathrm{dqd}, J=8.5,6.6,5.1 \mathrm{~Hz}, 1 \mathrm{H}), 4.59(\mathrm{dd}, J=12.6,4.3 \mathrm{~Hz}$, $1 \mathrm{H}), 4.53(\mathrm{dd}, J=12.5,5.5 \mathrm{~Hz}, 1 \mathrm{H}), 3.05(\mathrm{dt}, J=15.0,8.5 \mathrm{~Hz}$, $1 \mathrm{H}), 2.75$ (ddd, $J=15.0,7.0,5.2 \mathrm{~Hz}, 1 \mathrm{H}), 1.64(\mathrm{~d}, J=6.7 \mathrm{~Hz}$, $3 \mathrm{H}), 1.56$ (br t, $J=5.6 \mathrm{~Hz}, 1 \mathrm{H}) .13 \mathrm{C} \mathrm{NMR}\left(101 \mathrm{MHz}, \mathrm{CDCl}_{3}\right.$ ): $\delta 142.5,141.4,131.0(\mathrm{q}, J=32.4 \mathrm{~Hz}), 130.1,129.1,126.0$, $125.5,124.6(\mathrm{q}, J=3.8 \mathrm{~Hz}), 123.4(\mathrm{q}, J=4.0 \mathrm{~Hz}), 122.8,83.2$, 59.8, 34.0, 19.2. ${ }^{19} \mathrm{~F}$ NMR (376 $\left.\mathrm{MHz}, \mathrm{CDCl}_{3}\right): \delta$-62.7. HRMS (ESI+/TOF) Calcd for $\mathrm{C}_{13} \mathrm{H}_{14} \mathrm{~F}_{3} \mathrm{NO}_{3} \mathrm{Na}^{+}[\mathrm{M}+\mathrm{Na}]^{+}: 312.0818$. Found: 312.0819.

(Z)-4-(1-Hydroxy-5-nitrohex-2-en-2-yl)benzonitrile (4f). Compound $4 \mathbf{f}$ was prepared following the general procedure from cyclic carbonate 1f $(215.2 \mathrm{mg}, 1.0 \mathrm{mmol})$ and nitroethane. The product was purified by flash chromatography on silica gel using 30-40\% EtOAc/cyclohexane to afford the title compound (104.0 mg, $42 \%$ yield) as a yellowish oil. $R_{\mathrm{f}} 0.08$ (EtOAc/cyclohexane 3:7). IR (neat): 3494, 2989, 2939, 2898, 2227, 1605, 1544, 1504, 1449, 1389, 1360, 1312, 1008, 910, 830, $729 \mathrm{~cm}^{-1}$. ${ }^{1} \mathrm{H}$ NMR (400 MHz, $\mathrm{CDCl}_{3}$ ): $\delta 7.63-7.58(\mathrm{~m}, 2 \mathrm{H}), 7.54-7.48$ $(\mathrm{m}, 2 \mathrm{H}), 5.85(\mathrm{dd}, J=8.5,7.0 \mathrm{~Hz}, 1 \mathrm{H}), 4.73(\mathrm{dqd}, J=8.5,6.7$, $5.0 \mathrm{~Hz}, 1 \mathrm{H}), 4.56(\mathrm{~d}, J=12.6 \mathrm{~Hz}, 1 \mathrm{H}), 4.51(\mathrm{~d}, J=12.6 \mathrm{~Hz}$, $1 \mathrm{H}), 3.04$ (dt, $J=15.1,8.5 \mathrm{~Hz}, 1 \mathrm{H}), 2.75$ (ddd, $J=15.1,7.0,5.0$ $\mathrm{Hz}, 1 \mathrm{H}), 1.73$ (br s, 1H), 1.63 (d, $J=6.7 \mathrm{~Hz}, 3 \mathrm{H}) .{ }^{13} \mathrm{C} \mathrm{NMR}$ $\left(101 \mathrm{MHz}, \mathrm{CDCl}_{3}\right): \delta 145.2,142.2,132.4,127.3,127.2,118.9$, 111.3, 83.1, 59.4, 34.0, 19.2. HRMS (ESI+/TOF) Calcd for $\mathrm{C}_{13} \mathrm{H}_{14} \mathrm{~N}_{2} \mathrm{O}_{3} \mathrm{Na}^{+}[\mathrm{M}+\mathrm{Na}]^{+}:$269.0897. Found: 269.0907.

(Z)-5-Nitro-2-(thiophen-3-yl)hex-2-en-1-ol (4g). Compound $\mathbf{4 g}$ was prepared following the general procedure from 4-(thiophen-3-yl)-4-vinyl-1,3-dioxolan-2-one (196.2 mg, 1.0 $\mathrm{mmol}$ ) and nitroethane. The product was purified by flash chromatography on silica gel using 20-25\% EtOAc/cyclohexane to afford the title compound (159.3 mg, $70 \%$ yield) as a yellowish oil. Rf 0.09 (EtOAc/cyclohexane 2:8). IR (neat): 3567, 3381, 3108, 2988, 2938, 2897, 2248, 1678, 1544, 1449, 1388, 1360, 1312, 1004, 908, 861, 781, $728 \mathrm{~cm}^{-1}$. ${ }^{1} \mathrm{H}$ NMR $(500 \mathrm{MHz}$, $\left.\mathrm{CDCl}_{3}\right): \delta 7.33(\mathrm{dd}, J=3.0,1.4 \mathrm{~Hz}, 1 \mathrm{H}), 7.28(\mathrm{dd}, J=5.1,2.9$ $\mathrm{Hz}, 1 \mathrm{H}), 7.19$ (dd, $J=5.1,1.4 \mathrm{~Hz}, 1 \mathrm{H}), 5.85$ (dd, $J=8.3,7.3$ $\mathrm{Hz}, 1 \mathrm{H}), 4.68$ (dqd, $J=8.1,6.7,5.3 \mathrm{~Hz}, 1 \mathrm{H}), 4.51$ (d, $J=12.2$ $\mathrm{Hz}, 1 \mathrm{H}), 4.46(\mathrm{~d}, J=12.2 \mathrm{~Hz}, 1 \mathrm{H}), 2.99(\mathrm{dt}, J=15.0,8.3 \mathrm{~Hz}$, 1H), 2.71 (ddd, $J=15.0,7.2,5.4 \mathrm{~Hz}, 1 \mathrm{H}$ ), 1.73 (br s, $1 \mathrm{H}$ ), 1.60 $(\mathrm{d}, J=6.7 \mathrm{~Hz}, 3 \mathrm{H}) .{ }^{13} \mathrm{C} \mathrm{NMR}\left(126 \mathrm{MHz}, \mathrm{CDCl}_{3}\right): \delta 141.2$, 138.0, 126.1, 125.7, 122.8, 121.4, 83.3, 59.8, 33.7, 19.1. HRMS (ESI+/TOF) Calcd for $\mathrm{C}_{10} \mathrm{H}_{13} \mathrm{NO}_{3} \mathrm{SNa}^{+}[\mathrm{M}+\mathrm{Na}]^{+}: 250.0508$. Found: 250.0506.

(Z)-2-(Benzo[d][1,3]dioxol-5-yl)-5-nitrohex-2-en-1-ol (4h). Compound $4 \mathrm{~h}$ was prepared following the general procedure from 4-(benzo[d][1,3]dioxol-5-yl)-4-vinyl-1,3-dioxolan2-one (234.2 $\mathrm{mg}, 1.0 \mathrm{mmol}$ ) and nitroethane. The product was purified by flash chromatography on silica gel using 20-30\% EtOAc/cyclohexane to afford the title compound (162.4 mg, 61 $\%$ yield) as a yellowish oil. $R_{\mathrm{f}} 0.09$ (EtOAc/cyclohexane 2:8). IR (neat): 3567, 3401, 3068, 2989, 2897, 2779, 1720, 1606, $1545,1503,1486,1436,1388,1360,1326,1232,1110,1036$, 932, 891, 862, 808, $732 \mathrm{~cm}^{-1} .{ }^{1} \mathrm{H}$ NMR (400 MHz, $\left.\mathrm{CDCl}_{3}\right): \delta$ 6.91-6.84 (m, 2H), 6.77 (dd, $J=7.8,0.6 \mathrm{~Hz}, 1 \mathrm{H}), 5.95$ (s, 2H), $5.64(\mathrm{dd}, J=8.3,7.2 \mathrm{~Hz}, 1 \mathrm{H}), 4.68(\mathrm{dqd}, J=8.3,6.7,5.4 \mathrm{~Hz}$, $1 \mathrm{H}), 4.50(\mathrm{~d}, J=12.4 \mathrm{~Hz}, 1 \mathrm{H}), 4.46(\mathrm{~d}, J=12.4 \mathrm{~Hz}, 1 \mathrm{H}), 2.97$ $(\mathrm{dt}, J=15.0,8.2 \mathrm{~Hz}, 1 \mathrm{H}), 2.70(\mathrm{ddd}, J=15.0,7.2,5.4 \mathrm{~Hz}, 1 \mathrm{H})$, $1.60(\mathrm{~d}, J=6.7 \mathrm{~Hz}, 3 \mathrm{H}), 1.58$ (br s, $1 \mathrm{H}) .{ }^{13} \mathrm{C} \mathrm{NMR}(101 \mathrm{MHz}$, $\left.\mathrm{CDCl}_{3}\right): \delta 148.0,147.4,143.1,134.5,123.3,120.2,108.4$, 107.2, 101.3, 83.3, 59.9, 34.0, 19.0. HRMS (ESI+/TOF) Calcd for $\mathrm{C}_{13} \mathrm{H}_{15} \mathrm{NO}_{5} \mathrm{Na}^{+}[\mathrm{M}+\mathrm{Na}]^{+}:$288.0842. Found: 288.0840 .

(E)-2-(Furan-2-yl)-5-nitrohex-2-en-1-ol (4i). Compound 4i was prepared following the general procedure from 4-(furan-2yl)-4-vinyl-1,3-dioxolan-2-one $(83.4 \mathrm{mg}, 0.46 \mathrm{mmol})$ and nitroethane. The product was purified by flash chromatography on silica gel using 20-25\% EtOAc/cyclohexane to afford the 
title compound (33.3 mg, $34 \%$ yield) as a yellowish oil. $R_{\mathrm{f}} 0.11$ (EtOAc/cyclohexane 2:8). IR (neat): 3577, 3390, 3152, 3138, 3119, 2987, 2938, 2900, 1544, 1459, 1388, 1360, 1313, 1320, 1159, 1013, 905, 884, 805, $731 \mathrm{~cm}^{-1}$. ${ }^{1} \mathrm{H}$ NMR $(500 \mathrm{MHz}$, $\left.\mathrm{CDCl}_{3}\right): \delta 7.36(\mathrm{~d}, J=1.8 \mathrm{~Hz}, 1 \mathrm{H}), 6.42(\operatorname{app~d}, J=3.4 \mathrm{~Hz}, 1 \mathrm{H})$, 6.40 (dd, $J=3.4,1.8 \mathrm{~Hz}, 1 \mathrm{H}), 6.07$ (t, $J=8.0 \mathrm{~Hz}, 1 \mathrm{H}), 4.69$ $(\mathrm{dqd}, J=8.0,6.7,5.5 \mathrm{~Hz}, 1 \mathrm{H}), 4.47(\mathrm{~d}, J=12.3 \mathrm{~Hz}, 1 \mathrm{H}), 4.42$ $(\mathrm{d}, J=12.3 \mathrm{~Hz}, 1 \mathrm{H}), 3.03(\mathrm{dt}, J=15.0,8.2 \mathrm{~Hz}, 1 \mathrm{H}), 2.74$ (ddd, $J=15.0,7.5,5.6 \mathrm{~Hz}, 1 \mathrm{H}), 1.61$ (overlapped, $1 \mathrm{H}$ ), 1.61 (d, $J=$ $6.7 \mathrm{~Hz}, 3 \mathrm{H}) .{ }^{13} \mathrm{C}$ NMR $\left(126 \mathrm{MHz}, \mathrm{CDCl}_{3}\right): \delta 153.3,142.4$, $132.9,120.9$, 111.6, 106.9, 83.2, 58.2, 33.4, 19.1. HRMS (ESI+/TOF) Calcd for $\mathrm{C}_{10} \mathrm{H}_{13} \mathrm{NO}_{4} \mathrm{Na}^{+}[\mathrm{M}+\mathrm{Na}]^{+}: 234.0737$. Found: 234.0730.

(Z)-2-(Naphthalen-2-yl)-5-nitrohex-2-en-1-ol (4j). Compound $\mathbf{4} \mathbf{j}$ was prepared following the general procedure from 4 (naphthalen-2-yl)-4-vinyl-1,3-dioxolan-2-one (240.3 mg, 1.0 mmol) and nitroethane. The product was purified by flash chromatography on silica gel using $20 \%$ EtOAc/cyclohexane to afford the title compound (108.3 mg, $40 \%$ yield) as a yellowish oil. $R_{\mathrm{f}} 0.29$ (EtOAc/cyclohexane 3:7). IR (neat): 3574, 3390, 3057, 2939, 1543, 1448, 1388, 1360, 1013, 909, 802, 778, 730 $\mathrm{cm}^{-1} .{ }^{1} \mathrm{H}$ NMR $\left(500 \mathrm{MHz}, \mathrm{CDCl}_{3}\right): \delta 7.89-7.82(\mathrm{~m}, 2 \mathrm{H}), 7.80$ (app d, $J=8.3 \mathrm{~Hz}, 1 \mathrm{H}), 7.53-7.46(\mathrm{~m}, 2 \mathrm{H}), 7.43(\mathrm{dd}, J=8.3$, $7.0 \mathrm{~Hz}, 1 \mathrm{H}), 7.28(\mathrm{dd}, J=7.0,1.2 \mathrm{~Hz}, 1 \mathrm{H}), 5.60(\mathrm{dd}, J=8.3$, $7.1 \mathrm{~Hz}, 1 \mathrm{H}), 4.79-4.69$ (m, 1H), 4.58-4.48 (m, 2H), 3.12 (dt, $J$ $=14.7,8.3 \mathrm{~Hz}, 1 \mathrm{H}), 2.83(\mathrm{ddd}, J=14.8,7.0,5.4 \mathrm{~Hz}, 1 \mathrm{H}), 1.66$ $(\mathrm{d}, J=6.7 \mathrm{~Hz}, 3 \mathrm{H}), 1.53(\mathrm{br} s, 1 \mathrm{H}) .{ }^{13} \mathrm{C} \mathrm{NMR}(126 \mathrm{MHz}$, $\left.\mathrm{CDCl}_{3}\right): \delta 143.6,139.1,133.8,131.6,128.5,128.1,126.7$, 126.42, 126.40, 126.1, 125.43, 125.40, 83.3, 61.8, 33.9, 19.1 . HRMS (ESI+/TOF) Calcd for $\mathrm{C}_{16} \mathrm{H}_{17} \mathrm{NO}_{3} \mathrm{Na}^{+}[\mathrm{M}+\mathrm{Na}]^{+}$: 294.1101. Found: 294.1101.

Ethyl 6-hydroxy-5-(2-methoxyphenyl)-2-nitrohex-4-enoate (4l). Compound 41 was prepared following the general procedure B from cyclic carbonate $\mathbf{1 d}(220.2 \mathrm{mg}, 1.0 \mathrm{mmol})$ and ethyl nitroacetate. The product was purified by flash chromatography on silica gel using $20 \%$ EtOAc/cyclohexane to afford the title compound ( $247.2 \mathrm{mg}, 80 \%$ yield) as a yellowish oil. $R_{\mathrm{f}}$ 0.11 (EtOAc/cyclohexane 2:8). IR (neat): 3570, 2982, 2963, 2940, 2838, 1747, 1598, 1558, 1489, 1464, 1435, 1373, 1240, $1119,1050,1021,910,858,755,730 \mathrm{~cm}^{-1} .{ }^{1} \mathrm{H} \mathrm{NMR}(500 \mathrm{MHz}$, $\left.\mathrm{CDCl}_{3}\right): \delta 7.30-7.25(\mathrm{~m}, 1 \mathrm{H}), 7.08(\mathrm{dd}, J=7.5,1.8 \mathrm{~Hz}, 1 \mathrm{H})$, $6.96-6.91(\mathrm{~m}, 1 \mathrm{H}), 6.89(\mathrm{~d}, J=8.2 \mathrm{~Hz}, 1 \mathrm{H}), 5.58(\mathrm{t}, J=7.5 \mathrm{~Hz}$, $1 \mathrm{H}), 5.32(\mathrm{dd}, J=9.2,5.7 \mathrm{~Hz}, 1 \mathrm{H}), 4.39(\mathrm{dd}, J=12.5,7.1 \mathrm{~Hz}$, 1H), 4.33 (overlapped, $1 \mathrm{H}), 4.29$ (qd, $J=7.2,1.2 \mathrm{~Hz}, 2 \mathrm{H}), 3.85$ (s, 3H), 3.28 (ddd, $J=15.1,9.2,7.8 \mathrm{~Hz}, 1 \mathrm{H}), 3.16$ (ddd, $J=$ 15.3, 7.4, $5.7 \mathrm{~Hz}, 1 \mathrm{H}), 2.47(\mathrm{t}, J=6.5 \mathrm{~Hz}, 1 \mathrm{H}), 1.32(\mathrm{t}, J=7.1$ $\mathrm{Hz}, 3 \mathrm{H}) .{ }^{13} \mathrm{C} \mathrm{NMR}\left(126 \mathrm{MHz}, \mathrm{CDCl}_{3}\right): \delta 164.2,156.3,144.7$, 131.1, 130.3, 129.2, 125.4, 121.3, 110.7, 87.8, 63.2, 61.0, 55.7, 29.2, 14.0. Note: the NMR resonances reported correspond to the $(Z)$ isomer. HRMS (ESI+/TOF) Calcd for $\mathrm{C}_{15} \mathrm{H}_{19} \mathrm{NO}_{6} \mathrm{Na}^{+}$ $[\mathrm{M}+\mathrm{Na}]^{+}:$332.1105. Found: 332.1106.

(Z)-2-(2-Bromophenyl)-6-(furan-2-yl)-5-nitrohex-2-en-1-ol $(\mathbf{4 m})$. Compound $\mathbf{4 m}$ was prepared following the general procedure from cyclic carbonate $1 \mathrm{e}(269.1 \mathrm{mg}, 1.0 \mathrm{mmol})$ and nitroalkane 2c. The product was purified by flash chromatography on silica gel using 10-20\% EtOAc/cyclohexane to afford the title compound (173.0 mg, $47 \%$ yield) as a yellowish oil. $R_{\mathrm{f}}$ 0.32 (EtOAc/cyclohexane 2:8). IR (neat): 3581, 3407, 3119, 3055, 2919, 1548, 1506, 1468, 1431, 1370, 1145, 1010, 908, $728 \mathrm{~cm}^{-1} .{ }^{1} \mathrm{H}$ NMR (500 MHz, $\left.\mathrm{CDCl}_{3}\right): \delta 7.59-7.53(\mathrm{~m}, 1 \mathrm{H})$, $7.35(\mathrm{~d}, J=2.0 \mathrm{~Hz}, 1 \mathrm{H}), 7.31-7.24(\mathrm{~m}, 1 \mathrm{H}), 7.18-7.13(\mathrm{~m}, 2 \mathrm{H})$, $6.31(\mathrm{dd}, J=3.2,2.0 \mathrm{~Hz}, 1 \mathrm{H}), 6.17(\mathrm{~d}, J=3.3 \mathrm{~Hz}, 1 \mathrm{H}), 5.49$ (t,
$J=7.7 \mathrm{~Hz}, 1 \mathrm{H}), 4.91(\mathrm{tt}, J=8.2,5.5 \mathrm{~Hz}, 1 \mathrm{H}), 4.42(\mathrm{~d}, J=6.1$ $\mathrm{Hz}, 2 \mathrm{H}), 3.42(\mathrm{dd}, J=15.4,8.0 \mathrm{~Hz}, 1 \mathrm{H}), 3.24(\mathrm{dd}, J=15.5,6.0$ $\mathrm{Hz}, 1 \mathrm{H}), 3.04$ (dt, $J=15.1,8.5 \mathrm{~Hz}, 1 \mathrm{H}), 2.82$ (ddd, $J=15.1$, 7.1, $5.0 \mathrm{~Hz}, 1 \mathrm{H}), 1.72(\mathrm{t}, J=6.2 \mathrm{~Hz}, 1 \mathrm{H}) .{ }^{13} \mathrm{C} \mathrm{NMR}(126 \mathrm{MHz}$, $\left.\mathrm{CDCl}_{3}\right): \delta 149.1,144.9,142.5,142.1,132.7,131.2,129.2$, 127.5, 126.7, 122.4, 110.7, 108.3, 86.4, 60.8, 31.9, 31.8. HRMS (ESI+/TOF) Calcd for $\mathrm{C}_{16} \mathrm{H}_{16} \mathrm{BrNO}_{4} \mathrm{Na}^{+}[\mathrm{M}+\mathrm{Na}]^{+}: 388.0155$. Found: 388.0149 .

(Z)-7,7-Dimethoxy-5-nitro-2-(m-tolyl)hept-2-en-1-ol (4n). Compound $4 \mathbf{n}$ was prepared following the general procedure from cyclic carbonate $\mathbf{1 b}(204.2 \mathrm{mg}, 1.0 \mathrm{mmol})$ and nitroalkane 2e. The product was purified by flash chromatography on silica gel using 20-30\% EtOAc/cyclohexane to afford the title compound (263.1 mg, $85 \%$ yield) as a yellowish oil. $R_{\mathrm{f}} 0.12$ (EtOAc/cyclohexane 3:7). IR (neat): 3576, 3441, 2937, 2835, 2250, 1603, 1548, 1446, 1370, 1192, 1127, 1066, 1010, 909, $786,729,702 \mathrm{~cm}^{-1} .{ }^{1} \mathrm{H}$ NMR $\left(400 \mathrm{MHz}, \mathrm{CDCl}_{3}\right): \delta 7.25-7.16$ (m, 3H), 7.13-7.08 (m, 1H), $5.70(\mathrm{dd}, J=8.3,7.1 \mathrm{~Hz}, 1 \mathrm{H}), 4.76$ (tdd, $J=9.1,5.2,4.1 \mathrm{~Hz}, 1 \mathrm{H}), 4.52(\mathrm{dd}, J=14.5,12.6 \mathrm{~Hz}, 1 \mathrm{H})$, $4.48(\mathrm{~d}, J=12.6 \mathrm{~Hz}, 1 \mathrm{H}), 4.40(\mathrm{dd}, J=6.3,4.5 \mathrm{~Hz}, 1 \mathrm{H}), 3.36$ (s, 3H), 3.34 (s, 3H), 2.97 (dt, $J=14.9,8.4 \mathrm{~Hz}, 1 \mathrm{H}), 2.77$ (ddd, $J=15.0,7.1,5.2 \mathrm{~Hz}, 1 \mathrm{H}), 2.44(\mathrm{ddd}, J=14.7,9.2,4.5 \mathrm{~Hz}, 1 \mathrm{H})$, 2.35 (s, 3H), 2.04 (ddd, $J=14.8,6.3,4.1 \mathrm{~Hz}, 1 \mathrm{H}$ ), 1.72 (br s, 1H). ${ }^{13} \mathrm{C} \mathrm{NMR}\left(101 \mathrm{MHz}, \mathrm{CDCl}_{3}\right): \delta 143.9,140.3,138.3,128.6$, 128.5, 127.4, 123.73, 123.71, 102.1, 84.5, 59.8, 54.4, 54.0, 36.3, 33.1, 21.6. HRMS (ESI+/TOF) Calcd for $\mathrm{C}_{16} \mathrm{H}_{23} \mathrm{NO}_{5} \mathrm{Na}^{+}[\mathrm{M}+$ $\mathrm{Na}^{+}:$332.1468. Found: 332.1466.

(2Z,7Z)-5-Nitro-2-phenyl-8-(m-tolyl)nona-2,7-diene-1,9diol $(\boldsymbol{6} \boldsymbol{a})$. Compound 6a was prepared following the general procedure from cyclic carbonate $\mathbf{1 b}(72.3 \mathrm{mg}, 0.35 \mathrm{mmol})$ and mono-allylated nitroalkane 5a. The product was purified by flash chromatography on silica gel using 30-50 \% EtOAc/cyclohexane to afford the title compound (73.3 $\mathrm{mg}, 59 \%$ yield) as a yellowish foam. $R_{\mathrm{f}} 0.09$ (EtOAc/cyclohexane 4:6). IR (neat): 3575, 3376, 3080, 3056, 3028, 2920, 2249, 1602, 1546, $1491,1436,1371,1323,1220,1008,907,786,727,697,648$ $\mathrm{cm}^{-1} .{ }^{1} \mathrm{H}$ NMR $\left(500 \mathrm{MHz}, \mathrm{CDCl}_{3}\right): \delta 7.42-7.37(\mathrm{~m}, 2 \mathrm{H}), 7.36-$ $7.31(\mathrm{~m}, 2 \mathrm{H}), 7.31-7.27(\mathrm{~m}, 1 \mathrm{H}), 7.23(\mathrm{t}, J=7.5 \mathrm{~Hz}, 1 \mathrm{H}), 7.21-$ $7.16(\mathrm{~m}, 2 \mathrm{H}), 7.11(\mathrm{~d}, J=7.3 \mathrm{~Hz}, 1 \mathrm{H}), 5.75(\mathrm{t}, J=7.9 \mathrm{~Hz}, 1 \mathrm{H})$, $5.72(\mathrm{t}, J=7.9 \mathrm{~Hz}, 1 \mathrm{H}), 4.75(\mathrm{tt}, J=8.2,5.4 \mathrm{~Hz}, 1 \mathrm{H}), 4.54(\mathrm{~s}$, 2H), 4.53 (s, 2H), $3.06(\mathrm{dtd}, J=15.0,8.1,3.4 \mathrm{~Hz}, 2 \mathrm{H}), 2.87$ (dddd, $J=15.1,7.6,5.4,2.4 \mathrm{~Hz}, 2 \mathrm{H}), 2.35$ (s, 3H), 1.84 (br s, 2H). ${ }^{13} \mathrm{C}$ NMR $\left(126 \mathrm{MHz}, \mathrm{CDCl}_{3}\right): \delta 143.9,143.8,140.33$, 140.27, 138.4, 128.8, 128.7, 128.6, 128.0, 127.4, 126.7, 124.0, 123.7, 87.9, 59.94, 59.91, 32.3, 21.6. HRMS (ESI+/TOF) Calcd for $\mathrm{C}_{22} \mathrm{H}_{25} \mathrm{NO}_{4} \mathrm{Na}^{+}[\mathrm{M}+\mathrm{Na}]^{+}: 390.1676$. Found: 390.1681 .

(7Z)-2-(2-Bromophenyl)-5-nitro-8-(3-(trifluoromethyl)phenyl)nona-2,7-diene-1,9-diol $(\boldsymbol{6} \boldsymbol{b})$. Compound $\mathbf{6 b}$ was prepared following the general procedure from cyclic carbonate 1c (43.9 $\mathrm{mg}, 0.17 \mathrm{mmol}$ ) and mono-allylated nitroalkane $\mathbf{5 b}$. The product was purified by flash chromatography on silica gel using 30$40 \%$ EtOAc/cyclohexane to afford the title compound (35.0 $\mathrm{mg}, 41 \%$ yield) as a yellowish foam. $R_{\mathrm{f}} 0.07$ (EtOAc/cyclohexane 4:6). IR (neat): 3574, 3364, 3056, 2924, 1546, 1468, 1433, 1372, 1332, 1265, 1164, 1119, 1074, 1021, 800, 754, 700, 657 $\mathrm{cm}^{-1} .{ }^{1} \mathrm{H}$ NMR $\left(400 \mathrm{MHz}, \mathrm{CDCl}_{3}\right): \delta 7.66($ app s, $1 \mathrm{H}), 7.63$ $7.50(\mathrm{~m}, 3 \mathrm{H}), 7.46(\mathrm{t}, J=7.6 \mathrm{~Hz}, 1 \mathrm{H}), 7.30-7.25(\mathrm{~m}, 1 \mathrm{H}), 7.19$ $7.12(\mathrm{~m}, 2 \mathrm{H}), 5.83(\mathrm{dd}, J=8.4,7.0 \mathrm{~Hz}, 1 \mathrm{H}), 5.51(\mathrm{t}, J=7.7 \mathrm{~Hz}$, $1 \mathrm{H}), 4.78(\mathrm{tt}, J=8.3,5.3 \mathrm{~Hz}, 1 \mathrm{H}), 4.60(\mathrm{~d}, J=12.5 \mathrm{~Hz}, 1 \mathrm{H})$, $4.56(\mathrm{~d}, J=12.5 \mathrm{~Hz}, 1 \mathrm{H}), 4.48(\mathrm{~d}, J=13.3 \mathrm{~Hz}, 1 \mathrm{H}), 4.44(\mathrm{~d}, J$ $=13.3 \mathrm{~Hz}, 1 \mathrm{H}), 3.19-3.04(\mathrm{~m}, 2 \mathrm{H}), 2.97-2.86(\mathrm{~m}, 2 \mathrm{H}), 1.75(\mathrm{br}$ $\mathrm{s}, 2 \mathrm{H}) .{ }^{13} \mathrm{C} \mathrm{NMR}\left(101 \mathrm{MHz}, \mathrm{CDCl}_{3}\right): \delta 145.0,142.8,142.0$, 
$141.4,132.8,131.2,131.1$ (q, $J=32.5 \mathrm{~Hz}), 130.1,129.3,129.2$, $127.6,126.9,125.7,124.6(\mathrm{q}, J=3.9 \mathrm{~Hz}), 123.4(\mathrm{q}, J=3.5 \mathrm{~Hz})$, 122.5, 87.6, 61.0, 59.8, 32.3, 32.0. ${ }^{19} \mathrm{~F}$ NMR (376 MHz, $\left.\mathrm{CDCl}_{3}\right): \quad \delta$-62.7. HRMS (ESI+/TOF) Calcd for $\mathrm{C}_{22} \mathrm{H}_{21} \mathrm{BrF}_{3} \mathrm{NO}_{4} \mathrm{Na}^{+}[\mathrm{M}+\mathrm{Na}]^{+}:$522.0498. Found: 522.0521 .

(2Z,7Z)-2-(Benzo[d][1,3]dioxol-5-yl)-5-nitro-8-(thiophen3-yl)nona-2,7-diene-1,9-diol $(\boldsymbol{6} \boldsymbol{c})$. Compound $\mathbf{6 c}$ was prepared following the general procedure from 4-(benzo[d][1,3]dioxol5-yl)-4-vinyl-1,3-dioxolan-2-one (77.8 $\mathrm{mg}, 0.33 \mathrm{mmol})$ and mono-allylated nitroalkane 5c. The product was purified by flash chromatography on silica gel using 30-50\% EtOAc/cyclohexane to afford the title compound (76.4 mg, $57 \%$ yield) as a yellowish foam. $R_{\mathrm{f}} 0.10$ (EtOAc/cyclohexane 1:1). IR (neat): 3561, 3353, 3107, 2894, 1606, 1544, 1502, 1486, 1434, $1370,1325,1233,1102,1035,932,892,862,810,781,729$ $\mathrm{cm}^{-1} .{ }^{1} \mathrm{H}$ NMR $\left(400 \mathrm{MHz}, \mathrm{CDCl}_{3}\right): \delta 7.33(\mathrm{dd}, J=2.9,1.4 \mathrm{~Hz}$, $1 \mathrm{H}), 7.29(\mathrm{dd}, J=5.1,3.0 \mathrm{~Hz}, 1 \mathrm{H}), 7.19(\mathrm{dd}, J=5.0,1.4 \mathrm{~Hz}$, $1 \mathrm{H}), 6.88(\mathrm{t}, J=1.7 \mathrm{~Hz}, 1 \mathrm{H}), 6.85$ (overlapped, $1 \mathrm{H}), 6.77$ (d, $J$ $=7.9 \mathrm{~Hz}, 1 \mathrm{H}), 5.95(\mathrm{~s}, 2 \mathrm{H}), 5.87(\mathrm{t}, J=7.7 \mathrm{~Hz}, 1 \mathrm{H}), 5.65(\mathrm{t}, J$ $=7.7 \mathrm{~Hz}, 1 \mathrm{H}), 4.72(\mathrm{tt}, J=8.2,5.5 \mathrm{~Hz}, 1 \mathrm{H}), 4.50(\mathrm{~d}, J=12.4$ $\mathrm{Hz}, 1 \mathrm{H}), 4.47$ (d, $J=12.4 \mathrm{~Hz}, 1 \mathrm{H}), 4.46(\mathrm{~s}, 2 \mathrm{H}), 3.10-2.95(\mathrm{~m}$, 2H), 2.89-2.76 (m, 2H), 1.88 (br s, 2H). ${ }^{13} \mathrm{C} \mathrm{NMR} \mathrm{(101} \mathrm{MHz,}$ $\left.\mathrm{CDCl}_{3}\right): \delta 148.0,147.5,143.2,141.2,138.3,134.5,126.1$, $125.7,123.0,122.4,121.5,120.2$, 108.4, 107.2, 101.3, 87.9, 59.9, 59.8, 32.2, 32.0. HRMS (ESI+/TOF) Calcd for $\mathrm{C}_{20} \mathrm{H}_{21} \mathrm{NO}_{6} \mathrm{SNa}^{+}[\mathrm{M}+\mathrm{Na}]^{+}:$426.0982. Found: 426.0982 .

Synthesis of mono-allylated nitroalkanes. In a $5 \mathrm{~mL}$ vial, the respective cyclic carbonate $(1.0$ equiv. $),[\mathrm{Pd}(\text { allyl }) \mathrm{Cl}]_{2}(2.5$ mol \%) and DPEPhos (10 mol \%) were dissolved in $\mathrm{CH}_{3} \mathrm{NO}_{2}$ $(1.0 \mathrm{M})$. The vial was sealed with a septum and a needle was placed through the septum to release gaseous $\mathrm{CO}_{2}$. The mixture was stirred for $2-8 \mathrm{~h}$ at $\mathrm{rt}$ open to air. After that, the solvent was evaporated with a stream of $\mathrm{N}_{2}$ and the residue was purified by flash chromatography on silica gel to afford the corresponding mono-allylated nitroalkane. Compounds $\mathbf{5 a - 5 c}$ were prepared using this approach.

(Z)-5-Nitro-2-phenylpent-2-en-1-ol (5a). Compound 5a was prepared following the general procedure from cyclic carbonate 1a (190.2 mg, $1.0 \mathrm{mmol})$. The product was purified by flash chromatography on silica gel using 20-30\% EtOAc/cyclohexane to afford the title compound (110.1 mg, $53 \%$ yield) as a yellowish oil. $R_{\mathrm{f}} 0.16$ (EtOAc/cyclohexane 3:7). IR (neat): 3563, 3376, 3082, 3056, 3028, 2924, 2852, 1545, 1492, 1430, 1376, 1190, 999, 955, 766, $697 \mathrm{~cm}^{-1} .{ }^{1} \mathrm{H}$ NMR $(500 \mathrm{MHz}$, $\left.\mathrm{CDCl}_{3}\right): \delta 7.43-7.39(\mathrm{~m}, 2 \mathrm{H}), 7.37-7.33(\mathrm{~m}, 2 \mathrm{H}), 7.32-7.27(\mathrm{~m}$, $1 \mathrm{H}), 5.77$ (t, $J=7.6 \mathrm{~Hz}, 1 \mathrm{H}), 4.59(\mathrm{~s}, 2 \mathrm{H}), 4.53(\mathrm{t}, J=6.9 \mathrm{~Hz}$, 2H), 3.02 (q, $J=7.0 \mathrm{~Hz}, 2 \mathrm{H}), 1.54$ (br s, $1 \mathrm{H}) .{ }^{13} \mathrm{C}$ NMR (126 $\left.\mathrm{MHz} \mathrm{CDCl}_{3}\right): \delta 143.5,140.3,128.8,128.0,126.6,124.6,75.2$, 60.1, 26.6. HRMS (ESI+/TOF) Calcd for $\mathrm{C}_{11} \mathrm{H}_{13} \mathrm{NO}_{3} \mathrm{Na}^{+}[\mathrm{M}+$ $\mathrm{Na}]^{+}: 230.0788$. Found: 230.0790.

2-(2-Bromophenyl)-5-nitropent-2-en-1-ol (5b). Compound 5b was prepared following the general procedure from cyclic carbonate 1e $(269.1 \mathrm{mg}, 1.0 \mathrm{mmol})$. The product was purified by flash chromatography on silica gel using 20-30 \% EtOAc/cyclohexane to afford the title compound $(73.0 \mathrm{mg}, 25 \%$ yield) as an orange oil. $R_{\mathrm{f}} 0.14$ (EtOAc/cyclohexane 3:7). IR (neat): $3570,3384,3055,2919,1546,1467,1428,1376,1264,1192$, $1118,1023,752 \mathrm{~cm}^{-1} .{ }^{1} \mathrm{H}$ NMR $\left(400 \mathrm{MHz}, \mathrm{CDCl}_{3}\right): \delta 7.60-7.54$ $(\mathrm{m}, 1 \mathrm{H}), 7.31-7.26(\mathrm{~m}, 1 \mathrm{H}), 7.20-7.13(\mathrm{~m}, 2 \mathrm{H}), 5.52(\mathrm{t}, J=7.6$ $\mathrm{Hz}, 1 \mathrm{H}), 4.54(\mathrm{t}, J=6.8 \mathrm{~Hz}, 2 \mathrm{H}), 4.49(\mathrm{~d}, J=6.3 \mathrm{~Hz}, 2 \mathrm{H}), 3.03$ $(\mathrm{q}, J=7.0 \mathrm{~Hz}, 2 \mathrm{H}), 1.65(\mathrm{t}, J=6.3 \mathrm{~Hz}, 1 \mathrm{H}) .{ }^{13} \mathrm{C}$ NMR $(101$ $\left.\mathrm{MHz}, \mathrm{CDCl}_{3}\right): \delta 144.5,142.1,132.8,131.2,129.3,127.7$,
127.6, 122.5, 74.9, 61.0, 26.3. Note: the NMR resonances reported correspond to the $(Z)$ isomer. HRMS (ESI+/TOF) Calcd for $\mathrm{C}_{11} \mathrm{H}_{12} \mathrm{BrNO}_{3} \mathrm{Na}^{+}[\mathrm{M}+\mathrm{Na}]^{+}$: 307.9893. Found: 307.9891 .

(Z)-5-Nitro-2-(thiophen-3-yl)pent-2-en-1-ol (5c). Compound $\mathbf{5 c}$ was prepared following the general procedure from 4(thiophen-3-yl)-4-vinyl-1,3-dioxolan-2-one (196.2 mg, 1.0 $\mathrm{mmol}$ ). The product was purified by flash chromatography on silica gel using 20-30\% EtOAc/cyclohexane to afford the title compound (106.3 mg, $50 \%$ yield) as a yellowish oil. $R_{\mathrm{f}} 0.11$ (EtOAc/cyclohexane 3:7). IR (neat): 3565, 3376, 3106, 2916, 1544, 1428, 1376, 1336, 1267, 1178, 1001, 957, 861, 779, 734 $\mathrm{cm}^{-1} .{ }^{1} \mathrm{H}$ NMR $\left(500 \mathrm{MHz}, \mathrm{CDCl}_{3}\right): \delta 7.34(\mathrm{dd}, J=3.0,1.4 \mathrm{~Hz}$, $1 \mathrm{H}), 7.30(\mathrm{dd}, J=5.1,2.9 \mathrm{~Hz}, 1 \mathrm{H}), 7.20(\mathrm{dd}, J=5.2,1.4 \mathrm{~Hz}$, $1 \mathrm{H}), 5.89(\mathrm{t}, J=7.7 \mathrm{~Hz}, 1 \mathrm{H}), 4.54(\mathrm{~s}, 2 \mathrm{H}), 4.51(\mathrm{t}, J=6.8 \mathrm{~Hz}$, 2H), 2.99 (q, $J=7.0 \mathrm{~Hz}, 2 \mathrm{H}), 1.64$ (br s, 1H). ${ }^{13} \mathrm{C}$ NMR (126 $\left.\mathrm{MHz} \mathrm{CDCl}_{3}\right): \delta 141.2,138.0,126.2,125.7,123.1,121.4,75.1$, 59.9, 26.3. HRMS (ESI+/TOF) Calcd for $\mathrm{C}_{9} \mathrm{H}_{11} \mathrm{NO}_{3} \mathrm{SNa}^{+}[\mathrm{M}+$ $\mathrm{Na}^{+}:$236.0352. Found: 236.0356.

Synthesis of $3 \mathbf{p}, 4 \mathbf{k}$ and tris-allylated nitroalkanes $7 \mathbf{a}-c$. In a $5 \mathrm{~mL}$ vial, cyclic carbonate (1.0 equiv.), $\mathrm{Pd}(\mathrm{dba})_{2}(5 \mathrm{~mol}$ $\%)$, DPEPhos (10 mol \%) and nitroalkane (1.5 equiv.) were dissolved in MeCN (1.0 M). Then, DBU (10 mol \%) was added. The vial was sealed with a septum and a needle was placed through the septum to release gaseous $\mathrm{CO}_{2}$. The mixture was stirred for $2-8 \mathrm{~h}$ at $\mathrm{rt}$ open to air. After that, the solvent was evaporated with a stream of $\mathrm{N}_{2}$ and the residue was purified by flash chromatography on silica gel to afford the corresponding nitroalkane.

(Z)-5-Methyl-5-nitro-2-phenylhex-2-en-1-ol (3p). Compound 3p was prepared following the general procedure from cyclic carbonate $1 \mathrm{a}(38.0 \mathrm{mg}, 0.20 \mathrm{mmol})$ and 2-nitropropane. The product was purified by flash chromatography on silica gel using 20-30\% EtOAc/cyclohexane to afford the title compound (39.0 mg, $83 \%$ yield) as a yellowish oil. $R_{\mathrm{f}} 0.08$ (EtOAc/cyclohexane 2:8). IR (neat): 3575, 3405, 3057, 2987, 2930, 1533, 1493, 1468, 1445, 1396, 1372, 1347, 1261, 1137, 1019, 855, 766, $697 \mathrm{~cm}^{-1} .{ }^{1} \mathrm{H}$ NMR $\left(400 \mathrm{MHz}, \mathrm{CDCl}_{3}\right): \delta 7.43-7.27(\mathrm{~m}$, $5 \mathrm{H}), 5.72(\mathrm{t}, J=7.9 \mathrm{~Hz}, 1 \mathrm{H}), 4.55(\mathrm{~s}, 2 \mathrm{H}), 2.91(\mathrm{~d}, J=7.9 \mathrm{~Hz}$, $2 \mathrm{H}), 1.67$ (s, 6H), 1.49 (br s, 1H). ${ }^{13} \mathrm{C} \mathrm{NMR}\left(101 \mathrm{MHz}, \mathrm{CDCl}_{3}\right)$ : $\delta 143.8,140.5,128.7,127.9,126.7,123.9,88.3,59.9,39.5$, 26.0. HRMS (ESI+/TOF) Calcd for $\mathrm{C}_{13} \mathrm{H}_{17} \mathrm{NO}_{3} \mathrm{Na}^{+}[\mathrm{M}+\mathrm{Na}]^{+}$: 258.1101. Found: 258.1106.

(Z)-3-Methyl-5-nitro-2-phenylhex-2-en-1-ol (4k). Compound $4 \mathbf{k}$ was prepared following the general procedure from cyclic carbonate $1 \mathrm{~g}$ ( $40.8 \mathrm{mg}, 0.20 \mathrm{mmol})$ and nitroethane. The product was purified by flash chromatography on silica gel using 10-20\% EtOAc/cyclohexane to afford the title compound (30.1 mg, $64 \%$ yield) as a yellowish oil. $R_{\mathrm{f}} 0.15$ (EtOAc/cyclohexane 2:8). IR (neat): 3568, 3396, 3080, 3057, 3021, 2989, 2937, 2921, 2861, 1599, 1546, 1492, 1442, 1388, 1361, 1314, $1218,1118,1051,995,910,854,768,731,701 \mathrm{~cm}^{-1} .{ }^{1} \mathrm{H}$ NMR $\left(500 \mathrm{MHz}, \mathrm{CDCl}_{3}\right): \delta 7.38-7.32(\mathrm{~m}, 2 \mathrm{H}), 7.29-7.25(\mathrm{~m}, 1 \mathrm{H})$, 7.15-7.10 (m, 2H), 4.86 (dquint, $J=9.0,6.5 \mathrm{~Hz}, 1 \mathrm{H}), 4.34$ (d, $J=5.0 \mathrm{~Hz}, 2 \mathrm{H}), 3.13(\mathrm{dd}, J=14.1,9.0 \mathrm{~Hz}, 1 \mathrm{H}), 2.56(\mathrm{dd}, J=$ 14.1, $5.7 \mathrm{~Hz}, 1 \mathrm{H}), 1.64(\mathrm{~d}, J=6.6 \mathrm{~Hz}, 3 \mathrm{H}), 1.61$ (s, 3H), 1.36 (br t, $J=6.2 \mathrm{~Hz}, 1 \mathrm{H}) .{ }^{13} \mathrm{C}$ NMR $\left(126 \mathrm{MHz}, \mathrm{CDCl}_{3}\right): \delta 140.8$, $140.3,129.8,128.9,128.6,127.2,82.4,62.9,39.7,20.2,19.3$. HRMS (ESI+/TOF) Calcd for $\mathrm{C}_{13} \mathrm{H}_{17} \mathrm{NO}_{3} \mathrm{Na}^{+}[\mathrm{M}+\mathrm{Na}]^{+}$: 258.1101. Found: 258.1108 .

(2Z,7Z)-5-((Z)-4-Hydroxy-3-(m-tolyl)but-2-en-1-yl)-5-nitro-2-phenyl-8-(thiophen-3-yl)nona-2,7-diene-1,9-diol (7a). 
Compound 7a was prepared following the general procedure from 4-(thiophen-3-yl)-4-vinyl-1,3-dioxolan-2-one (26.1 mg, $0.13 \mathrm{mmol}$ ) and bis-allylated nitroalkane $6 \mathbf{6}$. The product was purified by flash chromatography on silica gel using 90-100\% $\mathrm{Et}_{2} \mathrm{O} /$ cyclohexane to afford the title compound (40.9 mg, $59 \%$ yield) as a yellowish foam. $R_{\mathrm{f}} 0.13\left(\mathrm{Et}_{2} \mathrm{O}\right)$. IR (neat): 3559, 3336, 3106, 3028, 2921, 1719, 1601, 1535, 1486, 1433, 1357, 1286, 1230, 1122, 1076, 1010, 847, 780, $697 \mathrm{~cm}^{-1} .{ }^{1} \mathrm{H}$ NMR $\left(500 \mathrm{MHz}, \mathrm{CDCl}_{3}\right): \delta 7.40-7.24(\mathrm{~m}, 7 \mathrm{H}), 7.24-7.14(\mathrm{~m}, 4 \mathrm{H})$, $7.10(\mathrm{~d}, J=7.4 \mathrm{~Hz}, 1 \mathrm{H}), 5.83(\mathrm{t}, J=7.4 \mathrm{~Hz}, 1 \mathrm{H}), 5.72-5.64(\mathrm{~m}$, 2H), 4.48 (d, $J=3.3 \mathrm{~Hz}, 4 \mathrm{H}), 4.43(\mathrm{~s}, 2 \mathrm{H}), 3.07$ (d, $J=7.4 \mathrm{~Hz}$, $6 \mathrm{H}), 2.47$ (s, 3H), 2.43 (br s, 3H), 2.34 (s, 3H). ${ }^{13} \mathrm{C}$ NMR (101 $\left.\mathrm{MHz}, \mathrm{CDCl}_{3}\right): \delta 144.0,141.3,140.4,140.3,138.6,138.5$, $131.1,129.0,128.9,128.8,128.7,128.0,127.4,126.7,126.1$, $125.8,125.7,123.8,123.0,122.7,121.6,121.4,94.4,59.93$, 59.90, 59.85, 34.8, 34.6, 30.5, 21.7. HRMS (ESI+/TOF) Calcd for $\mathrm{C}_{30} \mathrm{H}_{33} \mathrm{NO}_{5} \mathrm{SNa}^{+}[\mathrm{M}+\mathrm{Na}]^{+}:$542.1972. Found: 542.1982.

(2Z,7Z)-2-(Benzo[d][1,3]dioxol-5-yl)-5-((Z)-4-hydroxy-3(3-(trifluoromethyl)phenyl)but-2-en-1-yl)-5-nitro-8-(thiophen3-yl)nona-2,7-diene-1,9-diol (7b). Compound 7b was prepared following the general procedure from cyclic carbonate 1c (32.6 $\mathrm{mg}, 0.13 \mathrm{mmol}$ ) and bis-allylated nitroalkane $\mathbf{6 c}$. The product was purified by flash chromatography on silica gel using $\mathrm{Et}_{2} \mathrm{O}$ to afford the title compound (36.3 mg, $47 \%$ yield) as a yellowish foam. $\mathrm{R}_{\mathrm{f}} 0.06\left(\mathrm{Et}_{2} \mathrm{O}\right)$. IR (neat): 3642, 3559, 3350, 2954 , 2922, 1722, 1607, 1536, 1503, 1487, 1433, 1334, 1233, 1163, $1119,1075,1036,933,893,860,780,700 \mathrm{~cm}^{-1} ;{ }^{1} \mathrm{H}$ NMR $(500$ $\mathrm{MHz} \mathrm{CDCl}_{3}$ ): $\delta 7.63(\mathrm{~s}, 1 \mathrm{H}), 7.54($ app t, $\mathrm{J}=8.8 \mathrm{~Hz}, 2 \mathrm{H}), 7.42$ $(\mathrm{t}, \mathrm{J}=7.8 \mathrm{~Hz}, 1 \mathrm{H}), 7.30(\mathrm{dd}, \mathrm{J}=2.9,1.4 \mathrm{~Hz}, 1 \mathrm{H}), 7.28-7.24(\mathrm{~m}$, $1 \mathrm{H}), 7.18(\mathrm{dd}, \mathrm{J}=5.1,1.4 \mathrm{~Hz}, 1 \mathrm{H}), 6.89-6.81(\mathrm{~m}, 2 \mathrm{H}), 6.80$ $6.71(\mathrm{~m}, 1 \mathrm{H}), 5.93(\mathrm{~s}, 2 \mathrm{H}), 5.82(\mathrm{t}, \mathrm{J}=7.5 \mathrm{~Hz}, 1 \mathrm{H}), 5.75(\mathrm{t}, \mathrm{J}=$ $7.3 \mathrm{~Hz}, 1 \mathrm{H}), 5.60$ (t, J = 7.4 Hz, 1H), $4.48(\mathrm{~s}, 2 \mathrm{H}), 4.45$ (s, 2H), 4.43 (s, 2H), 3.12-3.01 (m, 6H), 2.55 (br s, 3H). 13C NMR (126 $\left.\mathrm{MHz}, \mathrm{CDCl}_{3}\right): \delta 148.0,147.5,143.3,142.6,141.5,141.3$, $138.3,135.9,134.6,130.9$ (q, J = 32.2 Hz), 130.1, 129.1, 126.1, 125.8, 125.6, 124.8, $124.5(\mathrm{q}, \mathrm{J}=3.7 \mathrm{~Hz}), 123.3(\mathrm{q}, \mathrm{J}=3.8 \mathrm{~Hz})$, 122.1, 121.5, 120.2, 108.5, 107.2, 101.3, 94.2, 59.8, 59.7, 59.6, 34.9, 34.8, 34.4. ${ }^{19} \mathrm{~F}$ NMR $\left(376 \mathrm{MHz}, \mathrm{CDCl}_{3}\right): \delta-62.7$. HRMS (ESI+/TOF) Calcd for $\mathrm{C}_{31} \mathrm{H}_{30} \mathrm{~F}_{3} \mathrm{NO}_{7} \mathrm{SNa}^{+}[\mathrm{M}+\mathrm{Na}]^{+}: 640.1587$. Found: 640.1582.

(2Z,7Z)-5-((Z)-4-Hydroxy-3-phenylbut-2-en-1-yl)-5-nitro2,8-diphenylnona-2,7-diene-1,9-diol (7c). Compound 7c was prepared following the general procedure from nitromethane $(10.8 \mu \mathrm{L}, 0.20 \mathrm{mmol})$ and cyclic carbonate $1 \mathbf{a}(117.9 \mathrm{mg}, 0.62$ mmol). The product was purified by flash chromatography on silica gel using 30-70 \% EtOAc/cyclohexane to afford the title compound (38.8 mg, $39 \%$ yield) as a yellowish foam. $R_{\mathrm{f}} 0.05$ (EtOAc/cyclohexane 1:1). IR (neat): 3568, 3326, 3055, 3024, 2953, 2922, 2851, 1598, 1534, 1492, 1443, 1353, 1013, 948, 847, 765, $695 \mathrm{~cm}^{-1} .{ }^{1} \mathrm{H}$ NMR $\left(400 \mathrm{MHz}, \mathrm{CDCl}_{3}\right): \delta 7.41-7.28$ $(\mathrm{m}, 15 \mathrm{H}), 5.70(\mathrm{t}, J=7.4 \mathrm{~Hz}, 3 \mathrm{H}), 4.54(\mathrm{~s}, 6 \mathrm{H}), 3.12(\mathrm{~d}, J=7.4$ $\mathrm{Hz}, 6 \mathrm{H}), 1.78$ (br s, 3H). ${ }^{13} \mathrm{C}$ NMR (101 MHz, $\left.\mathrm{CDCl}_{3}\right): \delta 144.1$, $140.4,128.8,128.0,126.7,123.0,94.4,59.9,34.8$. HRMS (ESI+/TOF) Calcd for $\mathrm{C}_{31} \mathrm{H}_{33} \mathrm{NO}_{5} \mathrm{Na}^{+}\left[\mathrm{M}+\mathrm{Na}^{+}: 522.2251\right.$. Found: 522.2254.

Synthesis of homoallylic amines 8a and 8b. In a $25 \mathrm{~mL}$ round-bottomed flask, the homoallylic nitroalkane substrate (1.0 equiv.) was dissolved in $\operatorname{EtOH}(0.15 \mathrm{M})$ under Ar. Then, 1 $\mathrm{M} \mathrm{HCl}(0.1 \mathrm{M})$ was added dropwise, followed by the addition of activated zinc dust (30 equiv.). The mixture was stirred at $\mathrm{rt}$ for $8 \mathrm{~h}$. The mixture was basified with $\mathrm{NaHCO}_{3}$ (aq, sat) and the solids were removed by filtration. The aqueous phase was extracted three times with EtOAc $(20 \mathrm{~mL})$. The combined organic layers were washed with brine, dried over $\mathrm{Na}_{2} \mathrm{SO}_{4}$, filtered and concentrated in vacuo. The crude product was purified by flash chromatography on silica gel to afford the corresponding homoallylic amine. See the SI for full analysis of these two compounds.

(Z)-5-amino-2-phenylhex-2-en-1-ol (8a). Compound 8a was prepared following the general procedure from homoallylic nitroalkane 3a $(66.4 \mathrm{mg}, 0.30 \mathrm{mmol})$. The product was purified by flash chromatography on silica gel using 10-20\% $\mathrm{MeOH} / \mathrm{CH}_{2} \mathrm{Cl}_{2}$ to afford the title compound $(42.5 \mathrm{mg}, 74 \%$ yield) as a yellowish oil. $R_{\mathrm{f}} 0.03\left(\mathrm{MeOH} / \mathrm{CH}_{2} \mathrm{Cl}_{2} 1\right.$ :9). IR (neat): 3344, 3283, 3080, 3055, 3025, 2959, 2925, 2871, 1727, 1597, 1493, 1445, 1374, 1343, 1009, 940, 765, $696 \mathrm{~cm}^{-1} .{ }^{1} \mathrm{H}$ NMR (500 MHz, CD $3 \mathrm{OD}): \delta 7.50-7.43(\mathrm{~m}, 2 \mathrm{H}), 7.34-7.27(\mathrm{~m}, 2 \mathrm{H})$, 7.26-7.19 (m, 1H), $5.90(\mathrm{t}, J=7.7 \mathrm{~Hz}, 1 \mathrm{H}), 4.53(\mathrm{~d}, J=12.1$ $\mathrm{Hz}, 1 \mathrm{H}), 4.50(\mathrm{~d}, J=12.1 \mathrm{~Hz}, 1 \mathrm{H}), 3.11$ (sext, $J=6.5 \mathrm{~Hz}, 1 \mathrm{H})$, 2.51-2.35 (m, 2H), $1.20(\mathrm{~d}, J=6.4 \mathrm{~Hz}, 3 \mathrm{H}) .{ }^{13} \mathrm{C}$ NMR $(126$ $\left.\mathrm{MHz}, \mathrm{CD}_{3} \mathrm{OD}\right): \delta 143.0,129.2,129.1,128.0,127.5,127.4$, 59.7, 48.3, 38.2, 22.2. HRMS (ESI+/TOF) Calcd for $\mathrm{C}_{12} \mathrm{H}_{18} \mathrm{NO}^{+}$ $[\mathrm{M}+\mathrm{H}]^{+}:$192.1383. Found: 192.1390.

(Z)-5-amino-3-methyl-2-phenylhex-2-en-1-ol (8b). Compound $\mathbf{8 b}$ was prepared following the general procedure from homoallylic nitroalkane $4 \mathbf{k}(32.6 \mathrm{mg}, 0.13 \mathrm{mmol})$. The product was purified by flash chromatography on silica gel using 10-20 $\% \mathrm{MeOH} / \mathrm{CH}_{2} \mathrm{Cl}_{2}$ to afford the title compound (48.7 mg, $79 \%$ yield) as a yellowish oil. $R_{\mathrm{f}} 0.06\left(\mathrm{MeOH} / \mathrm{CH}_{2} \mathrm{Cl}_{2} 1: 9\right)$. IR (neat): 3375, 3051, 3019, 2974, 2931, 2506, 2188, 1600, 1492, 1441, 1386, 1202, 1156, 1109, 1058, 993, 768, 720, $701 \mathrm{~cm}^{-1} .{ }^{1} \mathrm{H}$ NMR (500 MHz, CD $\left.{ }_{3} \mathrm{OD}\right): \delta 7.37-7.31(\mathrm{~m}, 2 \mathrm{H}), 7.28-7.23(\mathrm{~m}$, $1 \mathrm{H}), 7.23-7.19(\mathrm{~m}, 2 \mathrm{H}), 4.45(\mathrm{~d}, J=12.0 \mathrm{~Hz}, 1 \mathrm{H}), 4.25(\mathrm{~d}, J=$ $12.0 \mathrm{~Hz}, 1 \mathrm{H}), 3.53$ (sext, $J=6.9 \mathrm{~Hz}, 1 \mathrm{H}), 2.69(\mathrm{dd}, J=13.6,7.3$ $\mathrm{Hz}, 1 \mathrm{H}), 2.54(\mathrm{dd}, J=13.6,7.4 \mathrm{~Hz}, 1 \mathrm{H}), 1.64(\mathrm{~s}, 3 \mathrm{H}), 1.38(\mathrm{~d}$, $J=6.6 \mathrm{~Hz}, 3 \mathrm{H}) .{ }^{13} \mathrm{C} \mathrm{NMR}\left(126 \mathrm{MHz}, \mathrm{CD}_{3} \mathrm{OD}\right): \delta 143.3,140.9$, 132.3, 129.8, 129.2, 127.7, 63.2, 47.3, 40.3, 20.4, 19.3. HRMS (ESI+/TOF) Calcd for $\mathrm{C}_{13} \mathrm{H}_{19} \mathrm{NONa}^{+}[\mathrm{M}+\mathrm{Na}]^{+}: 228.1359$. Found: 228.1364.

\section{Synthesis of derivatives $8 \mathrm{c}$ and $8 \mathrm{~d}$.}

(Z)-5-nitro-2-phenylhex-2-en-1-yl benzoate (8c). In a flame-dried $25 \mathrm{~mL}$ round-bottomed flask, compound 3a (170.0 $\mathrm{mg}, 0.77 \mathrm{mmol})$ was dissolved in dry $\mathrm{CH}_{2} \mathrm{Cl}_{2}(2.3 \mathrm{~mL})$ under Ar. Then, pyridine ( $249 \mu \mathrm{L}, 3.07 \mathrm{mmol}$ ) was added dropwise and the mixture was cooled to $0{ }^{\circ} \mathrm{C}$. Thereafter, benzoyl chloride (107 $\mu \mathrm{L}, 0.92 \mathrm{mmol})$ was added dropwise, and the mixture was allowed to warm to $\mathrm{rt}$ and was stirred for $14 \mathrm{~h}$. The reaction was quenched with $1 \mathrm{M} \mathrm{HCl}$ and the phases were separated. The aqueous phase was extracted with $\mathrm{CH}_{2} \mathrm{Cl}_{2}(3 \times 10 \mathrm{~mL})$. The combined organic layers were washed with $\mathrm{NaHCO}_{3}$ (aq, sat) and brine $(1 \times 15 \mathrm{~mL})$, and were dried over $\mathrm{Na}_{2} \mathrm{SO}_{4}$, filtered and concentrated in vacuo. The crude product was purified by flash chromatography on silica gel using $5 \% \mathrm{Et}_{2} \mathrm{O} /$ hexane to afford compound 8c (178.9 mg, 72\% yield) as a colorless oil. $R_{\mathrm{f}} 0.26$ (Et ${ }_{2} \mathrm{O} /$ hexane 1:9). IR (neat): 3061, 3032, 2990, 2938, 2899, 1714, 1601, 1546, 1492, 1449, 1389, 1359, 1313, 1264, 1214, 1176, 1107, 1098, 1070, 1024, 958, 762, 738, 710, $697 \mathrm{~cm}^{-1} .{ }^{1} \mathrm{H}$ NMR (500 MHz, $\left.\mathrm{CDCl}_{3}\right): \delta$ 7.97-7.92 (m, 2H), 7.56-7.51 (m, 1H), 7.44-7.38 (m, 4H), 7.37-7.32 (m, 2H), 7.32-7.27 (m, 1H), $5.92(\mathrm{t}, J=7.6 \mathrm{~Hz}, 1 \mathrm{H}), 5.30(\operatorname{app~d}, J=12.5 \mathrm{~Hz}, 1 \mathrm{H}), 5.19$ (app $\mathrm{d}, J=12.5 \mathrm{~Hz}, 1 \mathrm{H}), 4.71(\mathrm{dqd}, J=8.1,6.7,5.5 \mathrm{~Hz}, 1 \mathrm{H}), 3.10$ (dt, $J=15.2,7.9 \mathrm{~Hz}, 1 \mathrm{H}), 2.88$ (ddd, $J=15.2,7.5,5.5 \mathrm{~Hz}, 1 \mathrm{H}$ ), $1.63(\mathrm{~d}, J=6.7 \mathrm{~Hz}, 3 \mathrm{H}) .{ }^{13} \mathrm{C} \mathrm{NMR}\left(126 \mathrm{MHz}, \mathrm{CDCl}_{3}\right): \delta 166.5$, $140.1,139.0,133.2$, 130.0, 129.8, 128.6, 128.5, 128.0, 127.1, 
126.5, 83.0, 61.5, 34.1, 19.1. HRMS (ESI+/TOF) Calcd for $\mathrm{C}_{19} \mathrm{H}_{19} \mathrm{NNaO}_{4}[\mathrm{M}+\mathrm{Na}]^{+}:$348.1206. Found: 348.1215.

(Z)-5-oxo-2-phenylhex-2-en-1-yl benzoate $(8 d) .{ }^{29}$ In an argon-flushed $25 \mathrm{~mL}$ round-bottomed flask, compound 8c $(65.1$ $\mathrm{mg}, 0.20 \mathrm{mmol}$ ) was dissolved in $\mathrm{MeOH}(3.0 \mathrm{~mL})$ under Ar. The solution was cooled to $0{ }^{\circ} \mathrm{C}$ and NaOMe $(137 \mu \mathrm{L}, 0.60$ mmol, $25 \%$ in $\mathrm{MeOH}$ ) was added. In a separate flask, $\mathrm{NH}_{4} \mathrm{OAc}$ (531.9 mg, $6.90 \mathrm{mmol})$ was dissolved in $\mathrm{H}_{2} \mathrm{O}(1.2 \mathrm{~mL})$ and $\mathrm{TiCl}_{3}(1.1 \mathrm{~mL}, 1.10 \mathrm{mmol}, 12 \%$ in $\mathrm{HCl})$ was added dropwise. After that, the mixture of $\mathrm{TiCl}_{3}$ was transferred with a cannula to the other reaction flask in 5 minutes. The mixture was stirred at $0{ }^{\circ} \mathrm{C}$ for $3 \mathrm{~h}$. Hereafter, the product was extracted with $\mathrm{Et}_{2} \mathrm{O}$ $(3 \times 10 \mathrm{~mL})$. The combined organic layers were washed with $\mathrm{NaHCO}_{3}(2 \times 10 \mathrm{~mL})$ and brine $(1 \times 10 \mathrm{~mL})$, dried over $\mathrm{MgSO}_{4}$, filtered and concentrated in vacuo. The crude product was purified by flash chromatography on silica gel using 5-15\% EtOAc/Hexane to afford compound 8d (31.1 mg, $53 \%$ yield) as a colorless oil. $R_{\mathrm{f}} 0.30$ (EtOAc/Hexane 2:8). IR (neat): 3060 , 3032, 2960, 2924, 2854, 1713, 1601, 1493, 1451, 1356, 1315, 1264, 1175, 1159, 1107, 1098, 1070, 1024, 952, 759, 710, 697 $\mathrm{cm}^{-1} .{ }^{1} \mathrm{H}$ NMR $\left(500 \mathrm{MHz}, \mathrm{CDCl}_{3}\right): \delta 7.99-7.91(\mathrm{~m}, 2 \mathrm{H}), 7.56$ $7.51(\mathrm{~m}, 1 \mathrm{H}), 7.51-7.48(\mathrm{~m}, 2 \mathrm{H}), 7.42-7.38(\mathrm{~m}, 2 \mathrm{H}), 7.37-7.32$ (m, 2H), 7.31-7.26 (m, 1H), $6.30(\mathrm{t}, J=7.2 \mathrm{~Hz}, 1 \mathrm{H}), 5.24(\mathrm{~s}$, 2H), 3.59 (d, J=7.2 Hz, 2H), 2.24 (s, 3H). ${ }^{13} \mathrm{C} \mathrm{NMR} \mathrm{(126} \mathrm{MHz,}$ $\left.\mathrm{CDCl}_{3}\right): \delta$ 205.5, 166.6, 140.2, 137.4, 133.2, 130.1, 129.8, 128.6, 128.5, 127.8, 126.4, 125.9, 62.0, 43.3, 30.1. HRMS (ESI+/TOF) Calcd for $\mathrm{C}_{19} \mathrm{H}_{18} \mathrm{NaO}_{3}[\mathrm{M}+\mathrm{Na}]^{+}$: 317.1148 . Found: 317.1149.

Crystallographic Studies. The measured crystal of $\mathbf{3 0}$ was stable under atmospheric conditions; nevertheless, it was treated under inert conditions immersed in perfluoro-polyether as protecting oil for manipulation. Data Collection: measurements were made on a Bruker-Nonius diffractometer equipped with an APPEX II 4K CCD area detector, a FR591 rotating anode with MoK $\alpha$ radiation, Montel mirrors and a Kryoflex low temperature device $\left(T=-173^{\circ} \mathrm{C}\right)$. Full-sphere data collection was used with $\omega$ and $\varphi$ scans. Programs used: Data collection Apex2 V2011.3 (Bruker-Nonius 2008), data reduction Saint+Version 7.60A (Bruker AXS 2008) and absorption correction SADABS V. 2008-1 (2008). Structure Solution: SHELXTL Version 6.10 (Sheldrick, 2000) was used. ${ }^{30}$ Structure Refinement: SHELXTL-97-UNIX VERSION.

Crystal data for homoallylic nitroalkane 30. $\mathrm{C}_{21.5} \mathrm{H}_{21} \mathrm{~N}_{2} \mathrm{O}_{5.5}, \quad M_{\mathrm{r}}=395.40$, monoclinic, $P 2(1) / \mathrm{n}, \quad a=$ 13.6502(8) ̊, $b=5.5638(3) \AA, c=25.477(2) \AA, \alpha=90^{\circ}, \beta=$ 93.556(6) ${ }^{\circ}, \gamma=90^{\circ}, V=1931.2(2) \AA^{3}, Z=4, \rho=1.360 \mathrm{mg} \cdot \mathrm{M}^{-3}$, $\mu=0.099 \mathrm{~mm}^{-1}, \lambda=0.71073 \AA, T=100(2) \mathrm{K}, F(000)=832$, crystal size $=0.20 \times 0.10 \times 0.05 \mathrm{~mm}, \theta(\min )=1.74^{\circ}, \theta(\max )=$ $27.80^{\circ}, 5097$ reflections collected, 5097 reflections unique, $\mathrm{GoF}=0.932, R_{1}=0.0418$ and $w R_{2}=0.0854[I>2 \sigma(I)], R_{1}=$ 0.0761 and $\mathrm{w} R_{2}=0.0970$ (all indices), $\mathrm{min} / \mathrm{max}$ residual density $=-0.202 / 0.245\left[\mathrm{e} \cdot \AA^{-3}\right]$. Completeness to $\theta\left(27.80^{\circ}\right)=88.6 \%$. CCDC number 1837204.

\section{ASSOCIATED CONTENT}

\section{Supporting Information}

The Supporting Information is available free of charge on the ACS Publications website at DOI: 10.1021/acsjoc.\#\#\# Catalysis screening data, NMR spectra for all known and new products (PDF), X-ray data for compound 30 (CIF).

\section{AUTHOR INFORMATION}

\section{Corresponding Author}

*E-mail: akleij@iciq.es

ORCID

Àlex Cristòfol: 0000-0001-6931-6423

Arjan W. Kleij: 0000-0002-7402-4764

\section{Notes}

The authors declare no competing financial interest.

\section{ACKNOWLEDGMENT}

We thank the Cerca program/Generalitat de Catalunya, ICREA, MINECO (CTQ2017-88920-P) and AGAUR (2017-SGR-232) for support.

\section{REFERENCES}

(1) (a) Itami, K.; Yoshida, J. Platform Synthesis: A Useful Strategy for Rapid and Systematic Generation of Molecular Diversity. Chem. Eur. J. 2006, 12 (15), 3966-3974. (b) Flynn, A. B.; Ogilvie, W. W. Stereocontrolled Synthesis of Tetrasubstituted Olefins. Chem. Rev. 2007, 107 (11), 4698-4745. (c) Negishi, E.; Huang, Z.; Wang, G.; Mohan, S.; Wang, C.; Hattori, H. Recent Advances in Efficient and Selective Synthesis of Di-, Tri-, and Tetrasubstituted Alkenes via Pd-Catalyzed Alkenylation-Carbonyl Olefination Synergy. Acc. Chem. Res. 2008, 41 (11), 1474-1485. (d) Shindo, M.; Mori, S. Torquoselective Olefination of Carbonyl Compounds with Ynolates: Highly Efficient Stereoselective Synthesis of Tetrasubstituted Alkenes. Synlett, 2008 (15), 2231-2243.

(2) (a) Ono, N. The Nitro Group in Organic Synthesis; John Wiley \& Sons: New York, 2001. (b) Ballini, R.; Bosica, G.; Fiorini, D.; Palmieri, A.; Petrini, M. Conjugate Additions of Nitroalkanes to ElectronPoor Alkenes: Recent Results. Chem. Rev. 2005, 105 (3), 933-972. (c) Ballini, R.; Petrini, M.; Rosini, G. Nitroalkanes as Central Reagents in the Synthesis of Spiroketals. Molecules 2008, 13 (2), 319-330.

(3) For selected reviews see: (a) Trost, B. M. Metal Catalyzed Allylic Alkylation: Its Development in the Trost Laboratories. Tetrahedron 2015, 71 (35), 5708-5733. (b) Butt, N. A.; Zhang, W. Transition Metal-Catalyzed Allylic Substitution Reactions with Unactivated Allylic Substrates. Chem. Soc. Rev. 2015, 44 (22), 7929-7967. (c) Qu, J.; Helmchen, G. Applications of Iridium-Catalyzed Asymmetric Allylic Substitution Reactions in Target-Oriented Synthesis. Acc. Chem. Res. 2017, 50 (10), 2539-2555. (d) Bayeh, L.; Tambar, U. K. Catalytic Asymmetric Intermolecular Allylic Functionalization of Unactivated Internal Alkenes. ACS Catal. 2017, 7 (12), 8533-8543.

(4) For the synthetic importance and utility of homoallylic amines see: (a) Ochoa Puentes, C.; Kouznetsov, V. Recent Advancements in the Homoallylamine Chemistry. J. Heterocyclic Chem. 2002, 39 (4), 595-614. (b) Ding, H.; Friestad, G. K. Asymmetric Addition of Allylic Nucleophiles to Imino Compounds. Synthesis 2005, (17), 2815-2829. (c) Shibasaki, M.; Kanai, M. Asymmetric Synthesis of Tertiary Alcohols and $\alpha$-Tertiary Amines via $\mathrm{Cu}$-Catalyzed $\mathrm{C}-\mathrm{C}$ Bond Formation to Ketones and Ketimines. Chem. Rev. 2008, 108 (8), 2853-2873. (d) Batey, R.; Ramadhar, T. Allylation of Imines and Their Derivatives with Organoboron Reagents: Stereocontrolled Synthesis of Homoallylic Amines. Synthesis 2011, (9), 1321-1346. (e) Gandhi, S.; List, B. Catalytic Asymmetric Three-Component Synthesis of Homoallylic Amines. Angew. Chem. Int. Ed. 2013, 52 (9), 2573-2576. (f) Niu, D.; Wan, L.; Tian, L.; Liu, J. Iridium-Catalyzed Asymmetric Umpolung Allylation of N-Fluorenyl Imines to Prepare 1,4-Disubstituted Homoallylic Amines. Synlett 2017, 28 (16), 2051-2056.

(5) (a) Rieck, H.; Helmchen, G. Palladium Complex Catalyzed Asymmetric Allylic Substitutions with Nitromethane: Enantioselectivities Exceeding 99.9\% ee. Angew. Chem. Int. Ed. Engl. 1996, 34 (23- 
24), 2687-2698. (b) Trost, B. M.; Surivet, J.-P. Diastereo- and Enantioselective Allylation of Substituted Nitroalkanes. J. Am. Chem. Soc. 2000, 122 (26), 6291-6292. (c) Trost, B. M.; Jiang, C. Atom Economic Asymmetric Creation of Quaternary Carbon: Regio- and Enantioselective Reactions of a Vinylepoxide with a Carbon Nucleophile. $J$. Am. Chem. Soc. 2001, 123 (51), 12907-12908. (d) Uozumi, Y.; Suzuka, T. $\pi$-Allylic C1-Substitution in Water with Nitromethane Using Amphiphilic Resin-Supported Palladium Complexes. J. Org. Chem. 2006, 71 (22), 8644-8646. (e) Ohmatsu, K.; Ito, M.; Kunieda, T.; Ooi, T. IonPaired Chiral Ligands for Asymmetric Palladium Catalysis. Nat. Chem. 2012, 4, 473-477. (f) Yang, X.-F.; Yu, W.-H.; Ding, C.-H.; Ding, Q.P.; Wan, S.-L.; Hou, X.-L.; Dai, L.-X.; Wang, P.-J. Palladium-Catalyzed Regio-, Diastereo-, and Enantioselective Allylation of Nitroalkanes with Monosubstituted Allylic Substrates. J. Org. Chem. 2013, 78 (13), 6503-6509. (g) Chaudhuri, S.; Bhunia, S.; Roy, A.; Das, M. K.; Bisai, A. Biomimetic Total Syntheses of Clavine Alkaloids. Org. Lett. 2018, 20 (1), 288-291.

(6) (a) Aleksandrowicz, P.; Piotrowska, H.; Sas, W. Palladium catalyzed C-allylation of nitroalkanes. Tetrahedron Lett. 1982, 38 (9), 1321-1327. (b) Maki, K.; Kanai, M.; Shibasaki, M. Pd-Catalyzed Allylic Alkylation of Secondary Nitroalkanes. Tetrahedron Lett. 2007, 63 (20), 4250-4257. (c) Grenning, A. J.; Tunge, J. A. Rapid Decarboxylative Allylation of Nitroalkanes. Org. Lett. 2010, 12 (4), 740-742. (d) Grenning, A. J.; Tunge, J. A. Deacylative Allylation of Nitroalkanes: Unsymmetric Bisallylation by a Three-Component Coupling. Angew. Chem. Int. Ed. 2011, 50 (7), 1688-1691. (e) Lang, S. B.; Locascio, T. M.; Tunge, J. A. Activation of Alcohols with Carbon Dioxide: Intermolecular Allylation of Weakly Acidic Pronucleophiles. Org. Lett. 2014, 16 (16), 4308-4311. (f) Chang, C.-Y.; Wu, Y.-K. Palladium-Catalyzed $\alpha$-Allylation of Secondary Nitroalkanes with Allylic Alcohols and Strategic Exploitation of Seebach's Reagent for the Total Synthesis of ( \pm )-Adalinine. J. Org. Chem. 2018, 83 (11), 6217-6224. For an intramolecular example see: (g) Genet, J. P.; Grisoni, S. Ergoline Precursors 2. An Efficient Synthesis of c Ring by Palladium (0) Catalyzed Reactions. Tetrahedron Lett. 1986, 27 (35), 4165-4168.

(7) Ognyanov, V.; Hesse, M. Palladium-Catalyzed Synthesis of 2Allyl-2-nitrocycloalkanones. Synthesis 1985, (6-7), 645-647.

(8) Chen, M. Z.; McLaughlin, M.; Takahashi, M.; Tarselli, M. A.; Yang, D.; Umemura, S.; Micalizio, G. C. Preparation of Stereodefined Homoallylic Amines from the Reductive Cross-Coupling of Allylic Alcohols with Imines. J. Org. Chem. 2010, 75 (23), 8048-8059.

(9) For selected seminal contributions: (a) Bando, T.; Harayama, H.; Fukazawa, Y.; Shiro, M.; Fugami, K.; Tanaka, S.; Tamaru, Y. Regioand Stereoselective Synthesis of 1,3-Hydroxyl Amines via PalladiumCatalyzed Carbonate-Carbamate Transformation with Unique Stereoselectivity: Synthesis of 3-Amino-4-penten-1-ols. J. Org. Chem. 1994, 59 (6), 1465-1474. (b) Ohmatsu, K.; Imagawa, N.; Ooi, T. Ligand-Enabled Multiple Absolute Stereocontrol in Metal-Catalysed Cycloaddition for Construction of Contiguous All-Carbon Quaternary Stereocentres. Nat. Chem. 2014, 6, 47-51. (c) Khan, A.; Zheng, R.; Kan, Y.; Ye, J.; Xing, J.; Zhang, Y. J. Palladium-Catalyzed Decarboxylative Cycloaddition of Vinylethylene Carbonates with Formaldehyde: Enantioselective Construction of Tertiary Vinylglycols. Angew. Chem. Int. Ed. 2014, 53 (25), 6439-6442. (d) Zhang, Y. J.; Yang, J. H.; Kim, S. H.; Krische, M. J. anti-Diastereo- and Enantioselective Carbonyl (Hydroxymethyl)allylation from the Alcohol or Aldehyde Oxidation Level: Allyl Carbonates as Allylmetal Surrogates. J. Am. Chem. Soc. 2010, 132 (13), 4562-4563.

(10) (a) Guo, W.; Martínez-Rodríguez, L.; Kuniyil, R.; Martin, E.; Escudero-Adán, E. C.; Maseras, F.; Kleij, A. W. Stereoselective and Versatile Preparation of Tri- and Tetra-Substituted Allylic Amine Scaffolds under Mild Conditions. J. Am. Chem. Soc. 2016, 138 (36), 1197011978. (b) Cai, A.; Guo, W.; Martínez-Rodríguez, L.; Kleij, A. W. Palladium-Catalyzed Regio- and Enantio-Selective Synthesis of Allylic Amines Featuring Tetrasubstituted Tertiary Carbons. J. Am. Chem. Soc. 2016, 138 (43), 14194-14197. (c) Guo, W.; Cai, A.; Xie, J.; Kleij, A. W. Asymmetric Synthesis of $\alpha, \alpha$-Disubstituted Allylic Amines through Palladium-Catalyzed Allylic Substitution. Angew. Chem. Int. Ed. 2017, 56 (39), 11797-11801.

(11) (a) Guo, W.; Martínez-Rodríguez, L.; Martín, E.; EscuderoAdán, E. C.; Kleij, A. W. Highly Efficient Catalytic Formation of (Z)-
1,4-But-2-ene Diols using Water as a Nucleophile. Angew. Chem. Int. Ed. 2016, 55 (37), 11037-11040. (b) Xie, J.; Guo, W.; Cai, A.; Escudero-Adán, E. C.; Kleij, A. W. Pd-Catalyzed Enantio- and Regioselective Formation of Allylic Aryl Ethers. Org. Lett. 2017, 19 (23), 6388-6391.

(12) Gómez, J. E.; Guo, W.; Kleij, A. W. Palladium-Catalyzed Stereoselective Formation of Substituted Allylic Thioethers and Sulfones. Org. Lett. 2016, 18 (23), 6042-6045.

(13) The preparation of (Z)-configured products from the allylation of nitroalkanes is not well-developed, see ref. 7.

(14) The isolated yield of $\mathbf{3 a}$ through column purification could not be improved despite several attempts.

(15) The low stereoselectivity towards $\mathbf{3 e}$ is ascribed to possible interference of the alcohol group of the nitroalkane with the catalytic species formed after decarboxylation of the VCC, see Scheme 1.

(16) Since a number of the resultant monoallylated nitroalkanes still incorporate an $\alpha$-acidic hydrogen, the products are susceptible towards double allylation. Such "over-allylation" has been observed by other authors, see for instance ref. 5a. Interestingly, Tunge and coworkers also reported a selective and sequential approach towards unsymmetrical bis-allylated nitroalkanes, see ref. $6 c$.

(17) We recently reported the cross-coupling of VCCs, see: W. Guo, W.; Kuniyil, R.; Gómez, J. E.; Maseras, F.; Kleij, A. W. A Domino Process towards Functionally Dense Quaternary Carbons through PdCatalyzed Decarboxylative Csp3-Csp3 Bond Formation. J. Am. Chem. Soc. 2018, 140 (11), 3981-3987.

(18) The group of Shibasaki reported that a basic additive is needed to boost the reactivity of the nitronate anion when secondary nitroalkanes are used, see ref. $6 \mathrm{~b}$.

(19) Benzoyl nitromethane is regarded as a synthetic equivalent to nitromethane. Intramolecular acyl transfer has been postulated as an operating mechanism, see: (a) Gao, Y.; Ren, Q.; Siau, W.-Y.; Wang, J. Asymmetric Organocatalytic Cascade Michael/Hemiketalization/Retro-Henry reaction of $\beta, \gamma$-Unsaturated Ketoesters with $\alpha$-Nitroketones. Chem. Commun. 2011, 47 (20) 5819-5821. (b) Lu, R.-J.; Yan, Y.-Y.; Wang, J.-J.; Du, Q.-S.; Nie, S.-Z.; Yan, M. Organocatalytic Asymmetric Conjugate Addition and Cascade Acyl Transfer Reaction of $\alpha$-Nitroketones. J. Org. Chem. 2011, 76 (15), 6230-6239. (c) Giorgi, G.; López-Alvarado, P.; Miranda, S.; Rodriguez, J.; Menéndez, J. C. Michael Additions in Aqueous Media: "On-Water" and "In-Water" Processes from $\alpha$-Nitro Ketones and Their Anions. Eur. J. Org. Chem. 2013, (7), 1327-1336.

(20) Yamamura, S.; Toda, M.; Hirata, Y. Modified Clemmensen Reduction: Cholestane. Org. Synth. 1973, 53, 86.

(21) Burk, R. M.; Roof, M. B. A Safe and Efficient Method for Conversion of 1,2- and 1,3-Diols to Cyclic Carbonates Utilizing Triphosgene. Tetrahedron Lett. 1993, 34 (3), 395-398.

(22) Piel, I.; Pawelczyk, M. D.; Hirano, K.; Fröhlich, R.; Glorius, F. A Family of Thiazolium Salt Derived N-Heterocyclic Carbenes (NHCs) for Organocatalysis: Synthesis, Investigation and Application in Cross-Benzoin Condensation. Eur. J. Org. Chem. 2011, (28), 54755484.

(23) Cai, S.; Zhao, X.; Wang, X.; Liu, Q.; Li, Z.; Wang, D. Z. Visible-light-promoted $\mathrm{C}-\mathrm{C}$ bond cleavage: photocatalytic generation of iminium ions and amino radicals. Angew. Chem. Int. Ed. 2012, 51 (32), 8050-8053.

(24) (a) Newkome, G. R.; Kim, H. J.; Moorefield, C. N.; Maddi, H.; Yoo, K.-S. Syntheses of New $1 \rightarrow(2+1)$ C-Branched Monomers for the Construction of Multifunctional Dendrimers. Macromolecules 2003, 36 (12), 4345-4354. (b) Rezazadeh, S.; Devannah, V.; Watson, D. A. Nickel-Catalyzed C-Alkylation of Nitroalkanes with Unactivated Alkyl Iodides. J. Am. Chem. Soc. 2017, 139 (24), 8110-8113.

(25) Manna, M. S.; Mukherjee, S. Organocatalytic Enantioselective Formal C(sp2)-H Alkylation. J. Am. Chem. Soc. 2015, 137 (1), 130133.

(26) Griesser, H.; Öhrlein, R.; Schwab, W.; Ehrler, R.; Jäger, V. 3Nitropropanal, 3-Nitropropanol, And 3-Nitropropanal Dimethyl Acetal. Org. Synth. 2000, 77, 236.

(27) Hamberger, H.; Stütz, P.; Schulz, G. Polare Äthylene II die Synthese von Nitro- und Amino-Acetaldehyddithioacetalen. Tetrahedron Lett. 1977, 18 (41), 3623-3624. 
(28) (a) Kai, Y.; Knochel, P.; Kwiatkowski, S.; Dunitz, J. D.; Oth, J. F. M.; Seebach, D.; Kalinowski, H.-O. Structure, Synthesis, and Properties of Some Persubstituted 1,2-Dinitroethanes. In Quest of Nitrocyclopropyl-Anion Derivatives. Helv. Chim. Acta 1982, 65 (1), 137-161. (b) Zhao, M.; Lu, W. Visible Light-Induced Oxidative Chlorination of Alkyl sp3 C-H Bonds with $\mathrm{NaCl} / \mathrm{Oxone}$ at Room Temperature. Org. Lett. 2017, 19 (17), 4560-4563.
(29) Ballini, R.; Petrini, M. The Nitro to Carbonyl Conversion (Nef Reaction): New Perspectives for a Classical Transformation. Adv. Synth. Catal. 2015, 357 (11), 2371-2402.

(30) G. M. Sheldrick, SHELXTL Crystallographic System, version 6.10; Bruker AXS, Inc.: Madison, WI, 2000. 